To Appear in the Ap.J. V.647, No.1 (Aug. 10, 2006)

Preprint typeset using $\mathrm{LAT}_{\mathrm{E} X}$ style emulateapj v. 21/08/00

\title{
THE CENTRAL ENGINES OF 19 LINERS AS VIEWED BY CHANDRA
}

\author{
Hélène M. L. G. Flohic ${ }^{1}$, Michael Eracleous ${ }^{1}$, George Chartas ${ }^{1}$, Joseph C. Shields ${ }^{2}$, \& \\ EDWARD C. MORAN ${ }^{3}$ \\ To Appear in the Ap.J. v.647, no.1 (Aug. 10, 2006)
}

\begin{abstract}
Using archival Chandra observations of 19 LINERs we explore the X-ray properties of their inner kiloparsec to determine the origin of their nuclear X-ray emission, to investigate the presence of an AGN, and to identify the power source of the optical emission lines . The relative numbers of LINER types in our sample are similar to those in optical spectroscopic surveys. We find that diffuse, thermal emission is very common and is concentrated within the central few hundred parsec. The average spectra of the hot gas in spirals and ellipticals are very similar to those of normal galaxies. They can be fitted with a thermal plasma $(k T \sim 0.5 \mathrm{keV})$ plus a power law (photon index of 1.3-1.5) model. There are on average 3 detected point sources in their inner kiloparsec with $10^{37}<L_{0.5-10 \mathrm{keV}}<10^{40} \mathrm{erg} \mathrm{s}^{-1}$. The average cumulative luminosity functions for sources in spirals and ellipticals are identical to those of normal galaxies. In the innermost circle of $2 . .5$ radius in each galaxy we find an AGN in 12 of the 19 galaxies. The AGNs contribute a median of $60 \%$ of the $0.5-10 \mathrm{keV}$ luminosity of the central $2 . .5$ region, they have luminosities of $10^{37}-10^{39} \mathrm{erg} \mathrm{s}^{-1}$ (Eddington ratios of $10^{-8}$ to $10^{-5}$ ). The ionizing luminosity of the AGNs is not enough to power the observed optical emission lines in this particular sample. Thus, we suggest that the lines are powered either by the mechanical interaction of an AGN jet (or wind) with the circumnuclear gas, or by stellar processes, such as photoionization by post-AGB stars from an old or intermediate-age population (in most cases) or by young stars (in a few cases).
\end{abstract}

Subject headings: galaxies: active - galaxies: nuclei - X-rays: galaxies

\section{INTRODUCTION}

In 1980, Heckman identified a class of galactic nuclei whose optical emission-line properties differ from those of classical active galactic nuclei (AGN) and $\mathrm{H}$ II regions. These objects have narrow emission lines whose relative intensities are indicative of a relatively low ionization state of the emitting gas. Heckman (1980) named these objects low-ionization nuclear emission-line regions (LINERs) and defined them using the ratios of the optical forbidden lines of oxygen: $[\mathrm{O}$ II $] \lambda 3727 /[\mathrm{O}$ III $] \lambda 5007>1$ and $\left[\begin{array}{ll}\mathrm{O} & \mathrm{I}\end{array}\right] 6300 /[\mathrm{O}$ III $] \lambda 5007>0.33$. Since the division is observationally motivated, any object satisfying one of the criteria and close to satisfying the other one is often called a LINER. Recognizing that this classification is somewhat arbitrary, Heckman (1980) noted the existence of "transition" objects whose spectra are intermediate between those of pure LINERs and H II regions.

The original idea proposed by Heckman (1980) to explain LINERs was that the emission lines are produced by shocks in the nucleus of the host galaxy. In fact, the optical spectrum arising from a shock-heated gas closely resembles the spectrum of LINERs (Dopita \& Sutherland 1995; Dopita et al. 1996). Similar emission could be produced by turbulent mixing layers between cold and hot ISM components (Filippenko 1996). Another possibility is that the lines originate in dense gas photo-ionized by X-rays (Ferland \& Netzer 1983; Halpern \& Steiner 1983). The ionizing source can be a low-luminosity AGN (LLAGN), an idea supported by the discovery of broad emission lines
(FWHM $>10^{3} \mathrm{~km} \mathrm{~s}^{-1}$ ) in the spectra of many LINERs (e.g., Filippenko \& Halpern 1984; Ho et al. 1997b; Stocrchi-Bergmann, Baldwin, \& Wilson 1993; Bower et al. 1996; Barth et al. 1999; Shields et al. 2000; Barth et al. 2001; Eracleous \& Halpern 2001). Alternatively, the source of ionizing photons could be the Wolf-Rayet or OB stars in a compact, young star cluster in the nucleus of the host galaxy (Terlevich \& Melnick 1985; Filippenko \& Terlevich 1992; Shields 1992; Barth \& Shields 2000). Yet another possibility is that post-AGB stars from an old stellar population provide the ionizing photons that power the LINER-like emission lines (Binette et al. 1994).

Recent spectroscopic surveys have shown that LINERs are very common in local galaxies, found in at least $30 \%$ of all galaxies and $50 \%$ of early-type spirals (see review by Ho 1996, and references therein). Since LINERs are so common, determining the nature of their power source is important and could have far-reaching implications. If most LINERs are genuine AGNs, they would be the most common type of AGN in the local universe and would represent the faint end of the AGN luminosity function. The study of LINERs could shed light on the evolution of AGN and their host galaxies since they are likely to represent the end state of quasar evolution. It would also set a lower limit to the frequency of accreting supermassive black holes (SMBHs) in galaxies. Moreover, the spectral energy distribution of LINERs is different from that of classical AGNs implying that, if they are AGNs, their mode of accretion might also be different (Lasota et al. 1996; Ho 1999). Suggested accretion modes are advection domi-

\footnotetext{
${ }^{1}$ Department of Astronomy \& Astrophysics, The Pennsylvania State University, 525 Davey Lab, University Park, PA 16802 e-mail: flohic, mce, chartas Castro.psu.edu

${ }^{2}$ Department of Physics \& Astronomy, Ohio University, 251B Clippinger Labs, Athens OH, 45701-2979 e-mail: shields@phy.ohiou.edu

3 Astronomy Department, Wesleyan University, Middletown, CT 06459 e-mail: ecm@astro.wesleyean.edu
} 
Flohic et al.

nated accretion flows (ADAFs; Narayan \& Yi 1994, 1995), convection-dominated accretion Flows (CDAF; Quataert \& Narayan 1999) and adiabatic inflow-outflow solutions (ADIOS; Blandford, \& Begelman 1999). If LINERs are powered by hot stars or stellar remnants, one can ask why their spectra are different from disk H II regions. Moreover, the presence of a central star cluster influences the chemical and dynamical evolution of the bulge of the galaxy (Boker 2000; Carollo 1999; Combes 2001), so the frequency of compact nuclear star clusters is of significant interest.

In view of the importance of determining the nature of the central engines of LINERs, we have undertaken the analysis of a large sample of archival Chandra observations of galaxies hosting LINERs. The X-ray morphology of the central kiloparsec of the nucleus at the angular resolution of Chandra can give a first hint to the power source. An unresolved hard X-ray source at the center of the galaxy is a good indication of an AGN, while a cluster of point sources and knotty diffuse emission suggests stellar phenomena. The high spatial resolution of Chan$d r a$ and its spectroscopic capabilities also make it possible to extract the spectra of the spatially-distinct components (point sources and diffuse emission) to confirm their nature and to evaluate their contribution to the energy budget. This is an important issue because some LINERs may be powered by a combination of an AGN and a starburst (hereafter "composites"; e.g., Veron et al. 1981, Moran et al. 1996, Fernandes et al. 2004).

Previous surveys with Chandra have studied the X-ray properties of LINERs with different techniques, purposes or samples than our study. Terashima \& Wilson (2003) used snapshot observations of LINERs with high radio fluxes in order to search for AGNs and determine their properties. Because of their short exposure times, they were able to detect AGNs with X-ray luminosities greater than $10^{38}-10^{39} \mathrm{erg} \mathrm{s}^{-1}$. Satyapal et al. (2005) and Dudik et al. (2005) used a combination of long and short Chandra observations to assess the presence of an AGN in LINERs and IRAS observations to determine the presence of star formation but they do not examine the energy bugdet of these sources. They did, however, determine that at least $50 \%$ of LINERs harbor a central hard X-ray source consistent with an AGN. Filho et al. (2004) find that a similar fraction of LINERs harbor AGNs by means of radio observations of 16 LINERs and Chandra observations of 8 of them. They focused on the properties of the nucleus and argued that LINERs are powered by an AGN, which is often undetected in the X-rays because the flux is highly absorbed. Eracleous et al. (2002) studied three LINERs addressing the same questions that we address here. Out of those three LINERs, one was powered by an AGN alone and two by stellar processes alone suggesting a diversity in the nature of the ionization sources of LINERs. In this paper we continue and extend the work of Eracleous et al. (2002). Some of the objects in our sample were studied individually using the same Chandra data that we use here. However, we repeat the analysis for the sake of uniformity and we add two important elements in our study: a detailed examination of the circumnuclear regions, and a careful consideration of the energy budget. The objects in our sample are unlike some of the notorious LINERs whose nature is clear-cut, but they are typical of the greater population of LINERs.

In this paper we present the results and conclusions of our study, organized as follows. The sample and initial data reduction procedures are described in $\S 2$. Model fits to the spectra of individual discrete sources and the average spectra of diffuse sources are described in $\S 3$. In $\S 4$ we analyze the properties of the point source populations collectively and in $\S 5$, we evaluate the power source of each galaxy from the sample individually. Finally, we summarize the results and discuss the implications in $\S 6$.

\section{TARGETS AND DATA SCREENING}

We examined observations of LINERs in the Chandra archive up to 2002 December and selected a sample of 19 galaxies hosting a pure LINER or a transition object (Ho et al. 1997a) based on their distance $(<25 \mathrm{Mpc})$ and their exposure time $(>15 \mathrm{ks})$. These selection criteria ensure good spatial resolution in the central kiloparsec of each galaxy, a high signal-to-noise ratio $(\mathrm{S} / \mathrm{N})$ and a low detection limit (the limiting $0.5-10 \mathrm{keV}$ point source luminosity is in the range $3.5 \times 10^{36}$ to $\left.3.4 \times 10^{37} \mathrm{erg} \mathrm{s}^{-1}\right)$. Most (14 out of 19) of the galaxies were observed for reasons unrelated to the presence of a LINER, thus the sample is largely unbiased with respect to the LINER properties. In $\S 6$, we use the distribution of LINER types in our sample, to argue that it is representative of the general population of LINERs.

The properties of the host galaxies and the exposure times are summarized in Table 1 . The LINER type follows the classification of Ho et al. (1997a) where L1 and L2 are "pure" LINERs. L1 have fairly strong narrow emission lines combined with weak broad $\mathrm{H} \alpha$ but no broad $\mathrm{H} \beta$ while L2 have only narrow emission lines. "Transition" objects with line ratios intermediate between those of $\mathrm{H}$ II regions and LINERs are referred to as T2. The distance is taken from Ho et al. (1997a) where they assume $H_{0}=75 \mathrm{~km} \mathrm{~s}^{-1} \mathrm{Mpc}^{-1}$. Column 5 gives the Galactic hydrogen column density $\left(N_{\mathrm{H}}\right)$ in the direction of the center of each galaxy (Dickey \& Lockman 1990). NGC 1553 was not included in the Ho et al. (1997a) sample, but was listed as a LINER by Phillips et al. (1986). Among the spiral galaxies, NGC 2681, NGC 3507, NGC 4314, NGC 5055 and NGC 7331 are viewed approximately face-on while NGC 4111 is approximately edge-on.

Table 1 also includes the $\mathrm{H} \alpha$ luminosities of the target galaxies, as well as the estimated black hole masses. The $\mathrm{H} \alpha$ luminosities were taken from from Ho et al. (1997a), who measured them from spectra obtained through a $2^{\prime \prime} \times 4^{\prime \prime}$ aperture. NGC 1553 is the only exception; its $\mathrm{H} \alpha$ luminosity was taken from Phillips et al. (1986). The black hole masses were estimated from the stellar velocity dispersion ${ }^{4}$ using the prescription of Tremaine et al. (2002). The derived black hole masses are between $10^{7}$ and $6.3 \times 10^{8} \mathrm{M}_{\odot}$, with an average of $1.2 \times 10^{8} \mathrm{M}_{\odot}$ and typical uncertainties of a factor of 2 (or \pm 0.3 dex; see discussion in Barth et al. 2002).

All the target galaxies were observed with the Advanced CCD Imaging Spectrometer (ACIS; Garmire et al. 2003). The nuclei of most galaxies were located near the S3 CCD

${ }^{4}$ Stellar velocity dispersions were taken from the Hypercat database, available electronically at http://www-obs.univ-lyon1.fr/hypercat. 
aimpoint, which did not suffer significant radiation damage during the early stages of the mission. Only NGC 3607 and NGC 3608 were on a different CCD: I2 and I0 respectively. The initial data screening was carried out with the CIAO software package $\mathrm{v} 3.2 .1^{5}$. It consisted of the selection of events with grades $0,2,3,4$ and 6 and the exclusion of events occurring during times of poor aspect solution and background flares. We also removed the $00^{\prime \prime} .5$ pixel randomization and checked for aspect-solution errors. We also applied a CTI correction to the data for the two ACIS-I sources. We corrected for pointing offsets using the appropriate information on the Chandra X-ray Center's web-site in order to improve the astrometry ${ }^{6}$.

After screening, we produced an event file of the region centered on the nucleus with a $1 \mathrm{kpc}$ radius. The coordinates of the stellar nucleus were determined from isophotal contours of infrared images obtained with 2MASS. When coordinates derived from radio measurements or dynamical measurements were available, they were compared with the 2MASS coordinates and we found that they were always in agreement within errors (except for the VLBA coordinates of NGC 4552, which were $1.25 \sigma$ away from the 2MASS coordinates). For reference we note that the typical astrometric uncertainty from 2MASS isophotal fitting is 1.225 , while the typical astrometric uncertainties from radio observations are 25 mas (VLA), 5 mas (VLBA), and 1.3 mas (VLBI). In comparison, the astrometric uncertainty of Chandra observations is 0.5 . The nucleus of all the galaxies is at most $7^{\prime \prime}$ away from the ACIS-S aimpoint, except for NGC 3607 and NGC 3608, whose nuclei are respectively $211^{\prime \prime}$ and $140^{\prime \prime}$ from the ACIS-I aimpoint.

\section{DATA ANALYSIS}

We begin the analysis of the data by constructing images of the central region of each galaxy, which we present in $§ 3.1$, below. This is followed by fitting models to the spectra of bright, discrete sources in the nuclei of the host galaxies (discussed in §3.2) and to the average spectra of diffuse emission in spiral and elliptical galaxies (presented in $\S 3.3)$.

\subsection{Images and Morphology}

From the processed event files, we produced images of a region of radius $1 \mathrm{kpc}$ around the center of each galaxy (hereafter, the "central kiloparsec") in two different bands: $0.5-2 \mathrm{keV}$ and $2-10 \mathrm{keV}$. We also produced hardness ratio maps by combining the soft $(S)$ and hard $(H)$ images in the following way: $H R=(H-S) /(H+S)$. For pixels which had no counts in either the soft or the hard band, we adopted a hardness ratio equal to the average of the image.

Figure 1 shows the image of the central kiloparsec of each target galaxy in the soft band $(0.5-2 \mathrm{keV})$ and the hard band $(2-10 \mathrm{keV})$ and the hardness ratio map. The morphology of each galaxy is summarized in Table 2 . The first column gives the name of the galaxy, while the second column gives the number of point sources that were identified by the wavdetect algorithm (Freeman et al. 2002) in the $0.5-10 \mathrm{keV}$ band. To prevent wavdetect from confusing fluctuations in diffuse emission with true discrete sources, we ran the algorithm on a $4 \times 4 \mathrm{kpc}$ region with a threshold of $10^{-6}$. An $\mathrm{S}$ in the third column indicates that a point source is exactly at the center of the galaxy in the $0.5-2 \mathrm{keV}$ energy band, while an $\mathrm{H}$ indicates that a point source in the $2-10 \mathrm{keV}$ energy band is at the galaxy center. The combination of an $\mathrm{S}$ and an $\mathrm{H}$ indicates that the central source is detected in both energy bands. To determine if a source is coincident with the center of a galaxy, we checked whether the Chandra source coordinates matched the nuclear coordinates (from 2MASS or radio observations) within the 1- $\sigma$ uncertainty region. The fourth column gives the same information as the third column but for the diffuse emission.

The morphology of the nuclear region as a function of energy can give a first hint for the power source of the LINERs. A hard central source suggests an AGN and a cluster of point sources or a knotty diffuse emission suggests stellar processes. When both are observed at the same time, this hints at a combination of a stellar processes and an AGN.

\subsection{Fits to Spectra of Discrete Sources}

We extracted the spectra of all the point sources using the ACIS Extract software (AE, Broos et al. 2002). AE uses polygonal extraction regions which approximate contours of the Chandra-ACIS point spread function (PSF). A PSF contour is selected such that the fraction of the PSF energy enclosed is approximately $95 \%$. The background was measured as close to the point sources as possible in order to decrease the contamination from diffuse emission, which is fairly common. Spectral fitting was carried out using XSPEC v.11.2.0x (Arnaud 1996). The spectra were truncated at $0.5 \mathrm{keV}$ at the low-energy end and between 2 and $10 \mathrm{keV}$, depending on the $\mathrm{S} / \mathrm{N}$, at the high-energy end. The spectral models were also modified by interstellar photoelectric absorption with cross sections as given by Morrison \& McCammon (1983).

Spectra of faint sources with 20-200 counts were binned adaptively by AE. These spectra were then fitted with an absorbed power-law model in order to determine the point source flux. Sources with less than 20 counts could not be fitted because of the small number of degrees of freedom. For these sources the luminosity was estimated by scaling a power law model $(\Gamma=1.8$, close to the median $\Gamma$ for the individual fits to the point sources) absorbed by the average Galactic column density $\left(N_{\mathrm{H}}=2 \times 10^{20} \mathrm{~cm}^{-2}\right)$ so that the predicted count rate equals the observed count rate. We chose this nominal value of $N_{\mathrm{H}}$ because it is an average Galactic column for the sample and a small variation in the $N_{\mathrm{H}}\left(<2 \times 10^{19} \mathrm{~cm}^{-2}\right)$ did not have an impact on the resulting X-ray flux. Using this model, we also estimated the lowest detectable $0.5-10 \mathrm{keV}$ point source luminosity for each galaxy, which we list in Table 2. The luminosities of all the discrete sources are used in our study of the luminosity functions in $\S 4$.

We estimated the uncertainties in our faint $(<200$ counts) X-ray point source luminosities by varying $\Gamma$ by \pm 0.2 for a power-law absorbed by the Galactic column. The $0.5-10 \mathrm{keV}$ luminosity then varied by $5-10 \%$. When

${ }^{5}$ Chandra Interactive Analysis of Observations (CIAO), http://cxc.harvard.edu/ciao/

${ }^{6}$ http://cxc.harvard.edu/cal/ASPECT/fix_offset/fix_offset.cgi 
Flohic et al.

varying $\Gamma$ by \pm 0.7 , the luminosity changes by $30 \%-40 \%$. When increasing the absorbing column density by one order of magnitude, the luminosities increased by $5-25 \%$. Increasing the absorbing column density by two orders of magnitude dramatically increased the luminosity by $100 \%-200 \%$. A small variation in the absorbing column density (20\%) did not have an impact on the X-ray luminosity estimate. Thus, based on the above estimates, and assuming that an absorbed power-law model provides an acceptable description of the spectrum, the typical uncertainty in the X-ray luminosities we derive is $\lesssim 30 \%$, unless the absorbing column density is grossly underestimated.

The spectra of sources with more than 200 counts were binned with a minimum of 20 counts per bin and different models were fitted to the spectra in order to determine the nature of the source and the luminosity. There is never more than one source per galaxy with more than 200 counts and it is usually the central point source. It is important to find the most apropriate model for the X-ray spectrum so as to determine the absorbing column reliably. Systematic errors in the absorbing column can lead to large systematic errors in the source luminosity. Thus, we have tested the following models:

- A blackbody spectrum (bb), with the temperature $(k T)$ and the normalization as free parameters.

- A comptonized blackbody spectrum (compbb, after Nishimura et al. 1986) with the seed blackbody photon temperature $\left(k T_{\text {seed }}\right)$, the electron temperature of the hot plasma $\left(k T_{\mathrm{e}}\right)$, the optical depth of the plasma $(\tau)$, and the normalization as free parameters.

- A spectrum from an accretion disk consisting of multiple blackbody components (diskbb, after Mitsuda et al. 1984; Makishima et al. 1986) with the temperature at the inner disk radius $\left(k T_{\text {in }}\right)$ and the normalization as free parameters.

- An emission spectrum from hot diffuse gas with up to three components at different temperatures (mekal, mekal2T, mekal3T; after Mewe et al. 1985, 1986; Kaastra 1992; Liedhal et al. 1995) with the plasma temperature $\left(k T_{i}, i=1,2,3\right)$, the metallicity $(Z$, initially set to solar), and the normalization as free parameters. The redshift was set to 0 since the galaxies are all nearby.

- A power-law spectrum (pow) with the photon index of the power-law $(\Gamma)$, and the normalization as free parameters.

- A spectrum from an accretion disk around a black hole in an X-ray binary Compton scattered from in a converging, plasma flow (bmc, after Titarchuk et al. 1997; Titarchuk \& Zannias 1998; Laurent \& Titarchuk 1999; Borozdin et al. 1999; Schrader \& Titarchuk 1999) with the temperature of the thermal photon source $\left(k T_{\text {seed }}\right)$, the index of the electron power-law energy distribution $(\alpha)$, the illumination parameter, and the normalization as free parameters.
We have also tested certain linear combinations of these models such as blackbody plus power law or mekal plus power law (as well as multiple mekal components at different temperatures).

These models can describe the spectra of X-ray binaries, accreting supermassive black holes or thermal diffuse emission depending on their luminosity and fit parameters. For low mass X-ray binaries, the shape of the spectrum depends on the spectral state (Barret 2001). In the high soft state, the spectrum can be modeled as a soft component (bb with $k T<1 \mathrm{keV}$ or diskbb with $k T_{\text {in }}$ between 0.5 and $1.5 \mathrm{keV}$ ) and a comptonized component (compbb) with $\tau \sim 5-15, k T_{\mathrm{e}} \sim 3 \mathrm{keV}$ and $k T_{\text {seed }}$ between 0.3 and 1.5 $\mathrm{keV}$ and with $L_{0.5-10 \mathrm{keV}}$ between $10^{37}$ and $10^{38} \mathrm{erg} \mathrm{s}^{-1}$. In the low hard hard state, the spectra low-mass X-ray binaries can be modeled as a soft component and a powerlaw tail but with with lower relative luminosities (and absolute luminosities below the detection limit in our sample galaxies). High-mass X-ray binaries have a hard spectrum with $L_{0.5-10 \mathrm{keV}}<10^{39} \mathrm{erg} \mathrm{s}^{-1}$ that can be modeled as a power-law of $\Gamma \sim 1.2$ (Persic \& Rephaeli 2001). Accreting supermassive black hole such as those found at the center of Seyfert galaxies are observed to have a power-law spectrum with $\Gamma \sim 2$. Their luminosities are typically greater than $10^{39} \mathrm{erg} \mathrm{s}^{-1}$ (see Mushotzky, Done, \& Pounds, 1993, and references therein), but less luminous AGNs are well known to exist, especially in LINERs.

Table 3 summarizes the two best-fitting models for the spectrum of the central object in each galaxy, or the brightest off-nuclear object. Fits with an acceptable value of the reduced $\chi^{2}$ but with unphysical parameters $\left(N_{\mathrm{H}}\right.$ lower than that listed in Table 1, unreasonable photon index or temperature) were not included in Table 3 . Column 2 of Table 3 gives the models that provided the best two fits and the values of the model parameters. Column 3 gives the $\chi^{2}$ and the number of degrees of freedom, and column 4 the luminosity calculated for the model in the $0.5-10 \mathrm{keV}$ range. For all these models, we assumed a solar metal abundance. In Figure 2, we show the best-fitting model for each spectrum (the first one listed for each object in Table 3) superposed on the data along with the post-fit residuals. When there were significant residuals, we tried to modify the metallicity using the values given by Terlevich $\&$ Forbes (2002) and letting it vary, but it never suppressed the residuals, nor did it improve the reduced $\chi^{2}$, and was not supported by a F-test; therefore we kept solar abundances. We explored the effects of assuming Solar abundance in objects with sub-solar or super-solar abundances by increasing and lowering the abundace and measuring the change in the other fit parameters. We found that the other fit parameters (column density, power-law index, temperature, luminosity) varied very slightly $(<5 \%)$ when the abundance increased or decreased by one order of magnitude.

\subsection{Diffuse Emission}

In $70 \%$ of the galaxies of our sample we find diffuse emission in the central kiloparsec. The frequent presence of diffuse emission was already noted by Halderson et al. (2001). In $87 \%$ of the galaxies that have diffuse emission, this emission contributes over $50 \%$ of the observed X-ray luminosity. The flux from the diffuse emission in each 
galaxy was not high enough to yield a high $\mathrm{S} / \mathrm{N}$ spectrum individually except for NGC 3628. Thus, we stacked the spectra from all the galaxies. First, we extracted the spectrum from a $1 \mathrm{kpc}$ radius around the center of each galaxy by carefully subtracting all the point sources and by choosing a background region free of diffuse emission and point sources. In the process of subtracting the point sources we also subtracted some diffuse emission, which makes our estimate of the diffuse emission luminosity a lower limit, strictly speaking. Since the number of point sources in each galaxy is small (3 on average), we estimate that this lower limit is not too far from the true luminosity. Before combining the spectra, we normalized them to a fiducial total count rate of $0.1 \mathrm{~s}^{-1}$ so that the result is a good representation of the average spectrum of this type of source. Then we stacked the spectra using the ftool addspec and propagated the errors appropriately. NGC 3607 and NGC 3608 were not included in the stacking procedure because they were observed with a different CCD and addspec requires that all the observations are made with the same CCD. We stacked separately the diffuse emission from spiral and elliptical galaxies. Figure 2 shows the resulting spectra.

The diffuse emission spectra were not well fitted by a two-temperature component plasma, instead they required a plasma model plus a power-law, with parameters as given in Table 3. The spectra themselves, with models superposed are shown in Figure 3. The model parameters are very similar between elliptical and spiral galaxies, $N_{\mathrm{H}} \approx(7-9) \times 10^{19} \mathrm{~cm}^{-2}, k t \approx 0.6 \mathrm{keV}, \Gamma=1.3-1.5$, with solar abundance. We also let the abundance vary as a free parameter, but the improvement in reduced $\chi^{2}$ was not significant according to the F-test. In $\S 4$ we estimate the average luminosity of unresolved X-ray binaries in the inner kiloparsec and we show that it is comparable to the luminosity of the power-law spectral component. We also fitted the diffuse emission spectrum of NGC 3628 independently and we fount that the two best models were either a two-temperature plasma model or a power law with parameters as given in Table 3 (the spectrum is shown in Fig. 2). The spectral parameters for this particular galaxy are in general agreement with what we find for the stacked spectra, above. We also note that David et al. (2005) studied the diffuse emission spectrum of one of the galaxies in our sample, NGC 3379, and found that it could be fitted with a similar model as our stacked spectra, above.

The plasma component of the diffuse emission has a similar temperature in both types of galaxies with a value that is slightly lower than what is observed in normal galaxies; $k T=0.6-0.7 \mathrm{keV}$ for spirals (Wang et al. 2001; Wang et al. 2003; Immler et al. 2003) and $k T=1 \mathrm{keV}$ for ellipticals (Forman et al. 1985; Trinchieri et al. 1986; Sivakoff et al. 2004). From the normalization constant of the XSPEC fit (after rescaling it to the average observed count rate), we can calculate the emission measure and then the density. We find that $n=6.2 \times 10^{-2} \mathrm{~cm}^{-3}$ for the elliptical galaxies and $n=4.8 \times 10^{-2} \mathrm{~cm}^{-3}$ for the spiral galaxies. These densities are comparable to those found in normal galaxies (e.g., Sivakoff et al. 2003; Soria et al. 2006a). From the stacked spectra, we can also deduce that the average X-ray luminosity of the diffuse emission (integrated over the inner kiloparsec) is $\left\langle L_{0.5-10 \mathrm{keV}}\right\rangle=5.3 \times 10^{38} \mathrm{erg} \mathrm{s}^{-1}$ for the ellipticals and $\left\langle L_{0.5-10 \mathrm{keV}}\right\rangle=8.5 \times 10^{38} \mathrm{erg} \mathrm{s}^{-1}$ for the spirals. Within uncertainties, the luminosities of the diffuse emission in spiral and elliptical galaxies are comparable to each other. We calculated the luminosity of the diffuse emission for each galaxy individually by scaling the model luminosity to the count rate for each galaxy. Table 4 summarizes the $0.5-2$ and $2-10 \mathrm{keV}$ luminosity of the diffuse emission within the central kiloparsec of each galaxy (Columns 2 and 3 ) and the $0.5-2$ and $2-10 \mathrm{keV}$ luminosity of the diffuse emission in the central 2.5 region (Columns 4 and 5). The last quantity was computed by integrating the model spectrum after scaling it to the count rate of each individual; we use this quantity in our evaluation of the power sources of individual galaxies in $\S 5$.

The sound speed of the hot gas is $c_{\mathrm{S}}=\left(\gamma k T / \mu m_{\mathrm{H}}\right)^{1 / 2} \approx$ $250 \mathrm{~km} \mathrm{~s}^{-1}$ (assuming solar composition, full ionization and $k T=0.5 \mathrm{keV} ; \gamma$ is the ratio of specific heats and $\mu$ is the mean molecular weight), slightly larger than the stellar velocity dispersions in these galaxies. The dynamical and cooling time scales of the hot gas are $\tau_{\mathrm{dyn}}=R / c_{s} \approx$ $10^{6}$ years and $\tau_{\text {cool }}=\frac{3}{2} n k T V / L_{\mathrm{X}} \approx 4 \times 10^{7}$ years, respectively (assuming that the gas is contained in a sphere of $R=200 \mathrm{pc}$ ). In view of these time scales and the fact that the sound speed is not considerably higher than the stellar velocity dispersion, it appears likely that the gas is bound to the host galaxy and its temperature is sustained by interaction with the stars. The gas may, in fact, originate in stellar mass loss. As David et al. (2005) note, the mass of hot gas in NGC 3379 is approximately $5 \times 10^{5} \mathrm{M}_{\odot}$ and it could have been supplied by stellar mass loss over a period of only $10^{7}$ years.

\section{POINT SOURCE POPULATION}

The LINERs in our sample have between zero and 12 point sources in their central kiloparsec (including candidate AGNs) with an average number of approximately 3 point sources and a most frequent value of two point sources. The point source detection limit for our sample varies between $3.5 \times 10^{36}$ and $3.4 \times 10^{37} \mathrm{erg} \mathrm{s}^{-1}$ in the $0.5-10 \mathrm{keV}$ band (see Table 2). The $0.5-10 \mathrm{keV}$ luminosity of the detected point sources varies between $1 \times 10^{37}$ and $1 \times 10^{40} \mathrm{erg} \mathrm{s}^{-1}$ with an average of $3.3 \times 10^{38} \mathrm{erg} \mathrm{s}^{-1}$.

In order to determine whether the point source populations of the central kiloparsec of LINERs differ from those of normal galaxies, we constructed luminosity functions (including point sources from all the galaxies in the sample but excluding candidate AGNs) and compared them to those of normal galaxies. The normal galaxy comparison sample comprises 6 galaxies with a wide range of Hubble types that have weak optical emission lines and have been observed intensively in the X-ray band (NGC 1637, NGC 3556, NGC 4365, NGC 4382, NGC 4631 and NGC 4697; Sivakoff et al. 2003; Sarazin et al. 2001; Wang et al. 2001; Immler et al. 2003).

We constructed the average luminosity functions for the elliptical galaxies and spiral galaxies separately using point source luminosities measured as described in $\$ 3.2$. We excluded candidate AGNs and truncated each luminosity function at the highest detection limit of galaxies in that category $\left(6.6 \times 10^{37}\right.$ and $3.4 \times 10^{37} \mathrm{erg} \mathrm{s}^{-1}$ for ellipticals and spiral respectively). There are 21 sources in the ellip- 
Flohic et al.

tical galaxy luminosity function and seven sources in the spiral galaxy luminosity function. The resulting cumulative luminosity functions are shown in Figure 4. We fitted a simple power-law through the cumulative luminosity function of the sources in spiral galaxies, which we derived from the differential luminosity function as follows:

$$
\begin{gathered}
\frac{d N}{d L} \equiv N(L) \equiv \frac{N_{0}}{L_{0}}\left(\frac{L}{L_{0}}\right)^{-\alpha} \\
\Rightarrow \quad N(>L) \equiv \int_{L}^{\infty} N(L) d L=N_{1}\left(\frac{L}{L_{0}}\right)^{-\beta},
\end{gathered}
$$

where $\alpha=1+\beta \neq 1$ and $N_{1}=N_{0} /(\alpha-1)$. In this convention $L_{0}$ is a fiducial luminosity and $N_{1}$ and $N_{0}$ are dimensionless constants (whose values depend on the choice of $L_{0}$ ).

The resulting index for spiral hosts, $\beta=0.8 \pm 0.2$, is in agreement with that found by Colbert et al. (2004) for normal spiral galaxies $(0.79 \pm 0.24)$. The cumulative luminosity function for the sources in elliptical galaxies is best fitted by a broken power-law with $\beta_{1}=0.40 \pm 0.01$ (for $L<L_{\text {break }}$ ), $\beta_{2}=1.4 \pm 0.2$ (for $L>L_{\text {break }}$ ) and $\log \left(L_{\text {break }} / \mathrm{erg} \mathrm{s}^{-1}\right)=38.7 \pm 0.1$. The value of $\beta_{2}$ is comparable to the value found by Colbert et al (2004) for normal elliptical galaxies (1.41 \pm 0.38$)$. But Colbert et al. (2004) do not mention a break in the luminosity function, although some of their luminosity functions seem to flatten in their Figure 3. Other authors find a break in the point-source luminosity function of normal elliptical galaxies (Sarazin et al. 2001; Blanton et al. 2001; Randall et al. 2004). On the other hand, Sivakoff et al. (2003) do not find a break in the luminosity function of the two elliptical galaxies NGC 4365 and NGC 4382. The break luminosity that some authors find is comparable to that from our luminosity function. This break could indicate that the nature of the XRB is different for sources above and below the break luminosity. Sarazin et al. (2001) suggested that the accretor of XRBs below the break luminosity is a neutron star, while the accretor of XRBs above the break luminosity is a black hole, based on the agreement of the break luminosity with the Eddington luminosity of a $1.4 \mathrm{M}_{\odot}$ neutron star. The value of $\beta_{1}$ does not match any value cited by Colbert et al. (2004) even though it is closest to the slope of the cumulative luminosity function of merger galaxies $(\beta=0.65)$. At any rate, the slope of the cumulative luminosity functions is well in the range of those for normal galaxies so LINERs do not appear to have an unusual XRB population.

NGC 3379 has 10 point sources in its inner $1 \mathrm{kpc}$ region, so we were able to construct a luminosity function for this individual galaxy. The cumulative luminosity function is well fitted by a power-law with $\beta=0.56 \pm 0.03$. This value is close to the value found by Colbert et al (2004) for starburst galaxies.

Using the above properties of the point source populations, we can investigate whether the power-law component in the diffuse emission spectra (see §3.3) can be due to the presence of unresolved X-ray binaries. To test this hypothesis, we extrapolated the luminosity function of equation (1) to very low luminosities and integrated it up to the minimum detectable luminosity of a resolved point source, i.e.,

$$
\begin{gathered}
L_{\mathrm{XP}}=\int_{L_{1}}^{L_{2}} L N(L) d L \\
=\frac{L_{0} N_{1} \beta}{1-\beta}\left(\frac{L_{2}}{L_{0}}\right)^{1-\beta}\left[1-\left(\frac{L_{1}}{L_{2}}\right)^{1-\beta}\right] \\
\approx \frac{L_{0} N_{1} \beta}{1-\beta}\left(\frac{L_{2}}{L_{0}}\right)^{1-\beta},
\end{gathered}
$$

where we have neglected the term in square brackets since $L_{2} \gg L_{1}$ and $\beta<1$. We apply equation (2) to elliptical and spiral galaxies using the lowest value from the luminosity functions of Figure 4 as the value of $L_{2}$. We find that for elliptical galaxies $\left(L_{0}=10^{38} \mathrm{erg} \mathrm{s}^{-1}\right.$, $\left.N_{1}=1.3, L_{2}=6.6 \times 10^{37} \mathrm{erg} \mathrm{s}^{-1}, \beta=0.82\right)$, the estimated unresolved point source luminosity $(0.5-10 \mathrm{keV})$ is $L_{\mathrm{XP}}=6 \times 10^{38} \mathrm{erg} \mathrm{s}^{-1}$, while for spiral galaxies $\left(L_{0}=10^{38} \mathrm{erg} \mathrm{s}^{-1}, N_{1}=54.3, L_{2}=3.4 \times 10^{37} \mathrm{erg} \mathrm{s}^{-1}\right.$, $\beta=0.40), L_{\mathrm{XP}}=2 \times 10^{39} \mathrm{erg} \mathrm{s}^{-1}$. These luminosities are certainly high enough to explain the hard X-ray component in the average diffuse emission spectra Also the spectra of the hard component have a power-law index of $\Gamma=1.2$, which is consistent with low hard state X-ray binaries.

\section{DISCUSSION OF INDIVIDUAL GALAXIES AND EVALUATION OF THE POWER SOURCE}

From Figure 1 and Table 2, there is no obvious trend in the morphology of the central kiloparsec of the galaxies. Some galaxies have a central point source, some do not. Some galaxies have diffuse emission and some do not. From Table 3 and Figure 2, the spectrum of the brightest sources in the galaxies are not all fitted by the same model and have a wide range of luminosity and model parameters. An evaluation of the power source of each individual galaxy is then required. To aid in our interpretation, we checked if the nuclei of the galaxies were observed at other wavelengths, especially in the radio and the UV. In general terms, a high X-ray radio loudness parameter ${ }^{7}$, defined as

$$
\log R_{\mathrm{X}}=\log \left[\frac{\nu L_{\nu}(5 \mathrm{GHz})}{L_{\mathrm{X}}(2-10 \mathrm{keV})}\right]>-4.5
$$

and a flat radio spectrum are indicative of a low luminosity AGN (hereafter LLAGN) (Terashima \& Wilson 2003) so the presence of a compact, high-brightness temperature radio core provides strong evidence of the presence of a LLAGN. An unresolved UV point source with non-stellar spectrum is also strong evidence for a LLAGN (Maoz et al. 1995), while an extended UV source is unlikely to be an AGN. If the flux of the UV point source varies over time, this indicates an AGN (Maoz et al. 2005). When a broad $\mathrm{H} \alpha$ was detected (i.e., the LINER is classified as L1), this demonstratates the presence of a LLAGN.

Finally, we checked whether our interpretation of the power source could be supported by the estimate of the age of the stellar population from population synthesis models,

7 This measure of radio loudness using X-ray luminosity $\left(R_{\mathrm{X}}\right)$ instead of the B luminosity $\left(R_{\mathrm{O}}\right)$ was introduced by Terashima \& Wilson $(2003)$ and the cut-off value of -4.5 was derived from the the optical cut-off $R_{\mathrm{O}}>10$ and the observed correlation between $R_{\mathrm{X}}$ and $R_{\mathrm{O}}$ for a sample of LLAGNs, Seyferts and PG quasars: $\log R_{O}=0.88 \log R_{\mathrm{X}}+5.0$. 
applied to specific galaxies. In cases where an AGN is not a likely power source, the emission lines could be powered either by photoionization from massive stars from a young stellar population or from post-AGB stars and the nuclei of young planetary nebulae associated with an old stellar population.

For LINERs with composite power sources (LLAGN plus stellar processes), we determined the contribution of the AGN to the central X-ray luminosity using the the spectrum from a circle of radius 2.5 at the center of the galaxy. We chose such an aperture because it has a similar projected area as the aperture used in the survey of Ho et al. (1997a) to measure the emission line luminosities. A radius of 2.5 corresponds to $120 \mathrm{pc}$ for a galaxy at $10 \mathrm{Mpc}$ and $300 \mathrm{pc}$ for a galaxy at $25 \mathrm{Mpc}$, i.e., it represents the very core the galaxies. We fitted this spectrum with a two-temperature plasma model plus a power-law. The power-law component parameters were fixed to those obtained during the analysis of the central point source; the two-temperature plasma models represents the diffuse emission. Then we calculated the $2-10 \mathrm{keV}$ luminosity of the central point source by integrating the power-law component, the $2-10 \mathrm{keV}$ luminosity of the full $2 . \prime 5$ central region from the two-temperature plasma plus power-law model, and thus the fraction of the X-ray luminosity in the central region contributed by the point source. The various $2-10 \mathrm{keV}$ luminosities are listed in Table 4 . We use this fractional contribution as a basis for classifying a LINER as a composite. When we use the $0.5-10 \mathrm{keV}$ band to calculate the contribution from the point source to the total central X-ray luminosity, the numbers change slightly without changing the conclusion about the dominant source (except in the case of NGC 4438 as explained in its individual description). All the luminosities are corrected for absorption.

NGC 1553 (L2/T2, S0) has three point sources in its central kiloparsec. The brightest one is exactly at the center of the galaxy and its spectrum is well fitted either by a two-temperature plasma model $\left(k T_{1}=0.14_{-0.09}^{+0.04} \mathrm{keV}, k T_{2}=18.0_{-10.4}^{+70} \mathrm{keV}\right)$ or a power-law $\left(\Gamma=1.2_{-0.1}^{+0.2}\right)$. The preference of a the two-temperature over a one-temperature plasma model and a power-law model is justified by the Ftest. Both of the above models give a luminosity of $L_{0.5-10 \mathrm{keV}}=1.5 \times 10^{40} \mathrm{erg} \mathrm{s}^{-1}$. With such a luminosity and spectral characteristics, this source is probably an AGN. This is supported by observations in the radio band: NGC 1553 is a weak radio source (10 mJy at $843 \mathrm{MHz}$; Harnett 1987). Assuming a radio spectral index of $p=-0.5$ (adopted as our default value hereafter; where $\left.S_{\nu} \propto \nu^{p}\right)$, we calculate the radio flux at $5 \mathrm{Ghz}$ to be $S_{5 \mathrm{Ghz}}=4.1 \mathrm{mJy}$ and $\log R_{\mathrm{X}}=-2.7$, which means that this source is radio loud. Blanton, Sarazin \& Irwin (2001) came to the same conclusion on the nature of the central source using the same data set but they estimated its luminosity to be slightly higher $\left(L_{0.3-10 \mathrm{keV}}=\right.$ $1.75 \times 10^{40}$ erg s $\left.\mathrm{s}^{-1}\right)$. We evaluated the luminosity of the central 2.5 region to be $L_{2-10 \mathrm{keV}}=$ $1.6 \times 10^{40} \mathrm{erg} \mathrm{s}^{-1}$. The luminosity of the AGN is evaluated by integrating the power-law that fits the data to be $L_{2-10 \mathrm{keV}}=1.5 \times 10^{40} \mathrm{erg} \mathrm{s}^{-1}$, which means that the AGN is the source of $92 \%$ of the $\mathrm{X}$-ray luminosity from the central region. This is consistent with estimates of the age of the stellar population that indicate that the stars in the nucleus of NGC 1553 are old (> 17 Gyr, Terlevich \& Forbes 2002; 10-15 Gyr, Longhetti et al. 2000).

NGC 2681 (L1, SB0/a) has three point sources within its central kiloparsec. The brightest point source is at the exact center and is detected in both the soft and hard bands. This is reminiscent of NGC 1553, except that NGC 2681 has more diffuse emission than NGC 1553. The spectrum of the central source is best fitted by a two-temperature plasma model or a power-law plus a single-temperature plasma model (see Table 3). The luminosity inferred from these models is $L_{0.5-10 \mathrm{keV}}=9.5 \times 10^{38} \mathrm{erg} \mathrm{s}^{-1}$. These fits do not exclude the possibility that the central source is a LLAGN since circumnuclear hot gas can give rise to the thermal component superposed on a power-law AGN spectrum. This source was detected in the radio waveband $\left(S_{\nu}=12.0 \mathrm{mJy}\right.$ at $1.49 \mathrm{GHZ}$; Condon 1987) leading to $\log R_{X}=-2.1$ (radio loud). A broad $\mathrm{H} \alpha$ emission line was detected in the optical (Ho et al. 1997b) suggesting that this source is a LLAGN. We estimated the total luminosity from the 2.5 central region to be $L_{2-10} \mathrm{keV}=$ $9.7 \times 10^{38} \mathrm{erg} \mathrm{s}^{-1}$ and the luminosity of the AGN to be $L_{2-10 \mathrm{keV}}=1.8 \times 10^{38} \mathrm{erg} \mathrm{s}^{-1}$ (by integrating the power-law component), which means that the potential AGN contributes only $\sim 20 \%$ of the total $\mathrm{X}$-ray luminosity of the central kiloparsec. This indicates that the contribution from stellar processes is dominant. The other $80 \%$ of the luminosity comes from three other point sources in the central $2 . .5$ region and diffuse emission. This is consistent with estimates that the solar metallicity stellar population of NGC 2681 was formed in a starburst episode about 1 Gyr ago (Gonzalez Delgado et al. 2004; Cappellari et al. 2001; Burnstein et al. 1988). An intermediate age $\left(10^{8}-10^{9}\right.$ years $)$ population has also been detected in the nucleus of this galaxy by (Cid Fernandes et al. 2005).

NGC 3379 (T2, E1) harbors a cluster of 10 point sources in its nucleus. One of these point sources (not the brightest one) is at the exact center of the galaxy. The spectrum of this source had only 89 counts, thus its luminosity was estimated by fitting an absorbed power-law: $L_{0.5-10 \mathrm{keV}}=6.3 \times 10^{37} \mathrm{erg} \mathrm{s}^{-1}$. An unresolved radio source of $S_{\nu}=0.7 \mathrm{mJy}$ has been detected at $5 \mathrm{GHz}$ at the center of this galaxy (Wrobel \& Heeschen 1991). We calculate $\log R_{X}=-2.3$, which makes this source radio loud and suggests that it is a LLAGN. The luminosity of the central $2 . .5$ region was evaluated to be $L_{2-10 \mathrm{keV}}=1.0 \times 10^{38} \mathrm{erg} \mathrm{s}^{-1}$ and the LLAGN has $L_{2-10 \mathrm{keV}}=1.7 \times 10^{37} \mathrm{erg} \mathrm{s}^{-1}$. The candidate LLAGN contributes $\sim 20 \%$ to the luminosity of the central 2.5 region, making this LINER a composite. However, age estimates indicate a stellar population of intermediate age, in the range of 6-9 Gyr (Terlevich \& Forbes 2002; Gregg et al. 2004). 
We were able to fit the brightest source in the nucleus with a number of models involving a power law and a soft component (diskbb, bb, mekal). The addition of the soft component to the simple power-law is not strongly justified by the F-test (chance probability between 14 and $44 \%$ depending on the model for the soft component; a plasma model to the power-law gives the smallest chance probability). The total luminosity of this source is $L_{0.5-10 \mathrm{keV}}=1.4 \times 10^{39} \mathrm{erg} \mathrm{s}^{-1}$. From its spectrum, luminosity, and off-center position, one can infer that this source is probably an X-ray binary (XRB).

NGC 3507 (L2, SBb) has two point sources in its central kiloparsec: one very faint (14 counts) close to the edge of the region and a central extended source embedded in a bright patch of diffuse emission. The central source had only 187 counts, which did not allow us to test a wide variety of spectral models. Instead we estimated its luminosity by fitting a simple mekal model (the photon index of the best-fitting power-law model was unrealistic) and we find $L_{0.5-10 \mathrm{keV}}=1.2 \times 10^{39} \mathrm{erg} \mathrm{s}^{-1}$ after correcting for absorption. A weak, extended radio source $\left(S_{\nu}=16 \mathrm{mJy}\right)$ is detected at $1.45 \mathrm{GHz}$ (Condon 1987), which suggests that this object is not a LLAGN. It could be a collection of SNRs producing a superbubble around it seen as the X-ray diffuse emission and radio extended source. The fact that the data could not be fitted with a realistic power-law index strengthen this idea. This indicates that the X-ray emission of this LINER is powered by stellar processes. This result is consistent with the estimate of the age of the stellar population, which indicates the presence of a young stellar component (10 ${ }^{6}$ years) (Gonzalez Delgado et al. 2004).

NGC 3607 (L2, S0) has two point sources embedded in diffuse emission, but none of them is at the center. The brightest point source did not have enough counts to justify fitting models to its spectrum; its luminosity was estimated to be $L_{0.5-10 \mathrm{keV}}=$ $1.4 \times 10^{39} \mathrm{erg} \mathrm{s}^{-1}$ by scaling the count rate to that expected from a power-law model. The absence of a central point source is a strong case against an AGN, in agreement with Terashima et al. (2002) who did not find evidence for an AGN from an X-ray spectrum obtained with ASCA. The point sources and the diffuse emission suggest that the $\mathrm{X}$-ray emission is powered by stellar processes. The age of the stellar population is estimated to be between 3.6 and 5.6 Gyr (Terlevich \& Forbes 2002; Proctor \& Sansom 2002).

NGC 3608 (L2, E2) has two point sources with the brightest one at the center, embedded in diffuse emission. The spectrum of the brightest source does not have enough counts to fit a variety of models to it but its luminosity was estimated to be $L_{0.5-10 \mathrm{keV}}=8.7 \times 10^{38} \mathrm{erg} \mathrm{s}^{-1}$. There is no detection of this source at $5 \mathrm{GHz}$ but only an upper limit of 0.5 mJy (Wrobel \& Heeschen 1991). We can then put a limit on the radio loudness: $\log R_{\mathrm{X}}<-2.7$. Thus this source might be radio loud, hence a candidate LLAGN. We note that this galaxy is the second most distant object in the sample and that the source is offset by 140 "'from the aim point of the CCD and the $50 \%$ encircled energy radius at $1.49 \mathrm{keV}$ at this off-axis location is 1 .' 5 , i.e. a large fraction of the 2 .' 5 radius region used to determine the contribution to the central X-ray luminosity. Also such a large PSF increases the chance of source confusion and the chance that this is a multiple source. The age of the stellar population in NGC 3608 has been estimated to be between 9 and 10 Gyr (Terlevich \& Forbes 2002; Proctor \& Sansom 2002). We estimate that the luminosity of the central $2^{\prime \prime} .5$ is $L_{2-10 \mathrm{keV}}=1 \times$ $10^{39} \mathrm{erg} \mathrm{s}^{-1}$ and the luminosity of the central source is $L_{2-10 \mathrm{keV}}=6 \times 10^{38} \mathrm{erg} \mathrm{s}^{-1}$ so the hypothetical AGN contributes $60 \%$ of the X-ray luminosity.

NGC 3628 (T2, Sb) has five point sources in its central kiloparsec including a bright point source $1 \mathrm{kpc}$ away from the center. There is no point source at the center of the galaxy, but a central patch of diffuse emission. The spectrum of the diffuse emission is well fitted by a variety of models with a two-temperature plasma or a power-law giving the best fits. The resulting luminosity is $\left(L_{0.5-10 \mathrm{keV}}=4.6 \times 10^{38} \mathrm{erg} \mathrm{s}^{-1}\right)$. An extended radio source was detected at the center of NGC 3628 (Condon 1987), which could indicate a SN-driven superbubble. This galaxy is believed to be a starburst galaxy due to the presence of numerous XRBs (Strickland et al. 2001). There is no study of the stellar population that gives an estimate of the age. The absence of a LLAGN, the point sources and the diffuse emission suggest that the X-ray emission of this LINER is powered by stellar processes (perhaps a young starburst).

NGC 4111 (L2, S0) has only one central point source with a hard X-ray spectrum. There is also a fair amount of diffuse emission. The spectrum extracted from the central source does not have enough counts to justify fitting models to it but the luminosity can be estimated to be $L_{0.5-10 \mathrm{keV}}=4.4 \times 10^{39} \mathrm{erg} \mathrm{s}^{-1}$ and $L_{2-10 \mathrm{keV}}=3.7 \times 10^{39} \mathrm{erg} \mathrm{s}^{-1}$. There is a detection of a central source in the radio with $S_{5 \mathrm{GHz}}=$ 2.3 mJy (Wrobel \& Heeschen 1991), which means that $\log R_{\mathrm{X}}=-3$. The radio loudness, the central position of the source and the hard X-ray spectrum suggest that this source is a LLAGN. We evaluated the luminosity of the central 2.5 region to be $L_{2-10 \mathrm{keV}}=4.8 \times 10^{39} \mathrm{erg} \mathrm{s}^{-1}$, meaning that the point source contributes to $\sim 77 \%$ of the Xray luminosity. The age of the stellar population of NGC 4111 was estimated to less than 1 Gyr (Gregg 1989).

NGC 4125 (T2, E6) has only one hard point source at its center surrounded by diffuse emission. This point source did not have enough counts to justify fitting various models to its spectrum, but its luminosity was estimated to be $L_{0.5-10 \mathrm{keV}}=9 \times 10^{38} \mathrm{erg} \mathrm{s}^{-1}$. 
This source was not detected in the radio but a limit was set: $0.5 \mathrm{mJy}$ at $5 \mathrm{GHz}$ (Wrobel\& Heeschen 1991). This means that $\log R_{\mathrm{X}}<-2.7$ so this source could be radio loud. Due to its central position and possible radio loudness, this source could be a LLAGN. We estimate that the luminosity of the central 2 .! 5 region is $L_{2-10 \mathrm{keV}}=6.7 \times 10^{38} \mathrm{erg} \mathrm{s}^{-1}$ and the LLAGN has $L_{2-10 \mathrm{keV}}=5.4 \times 10^{38} \mathrm{erg} \mathrm{s}^{-1}$, i.e. $\sim 80 \%$ of the X-ray luminosity. The remaining X-ray luminosity is contributed by the diffuse emission.

NGC 4314 (L2, SBa) has no point sources, just sparse soft diffuse emission. NGC 4314 is one of the nearest examples of galaxies hosting a circumnuclear ring of star formation. Its diameter is $10^{\prime \prime}$, which falls within the central kiloparsec. The age of the stellar population in the nuclear ring has been estimated to be very young (1-15 Myr, Benedict et al. 2002). So the X-ray emission of this LINER is very likely powered by young stars.

NGC 4374 (L2, E1) has four point sources in its nucleus. The brightest one is at the center and has a hard X-ray spectrum. There is also significant soft, knotty, diffuse emission, although the knots could be embedded point sources. There are many models that give good fits, in particular a power-law and a plasma model plus a power-law. The F-test justifies the addition of a plasma component to the power-law (chance probability $=4 \times 10^{-4}$ ). The luminosity is $L_{0.5-10 \mathrm{keV}}=7.7 \times 10^{39} \mathrm{erg} \mathrm{s}^{-1}$. The stellar population of NGC 4374 is quite old (13.7 Gyr, Proctor \& Sansom 2002; 11.8 Gyr, Terlevich \& Forbes 2002). Radio images of NGC 4374 show structures resembling jets (De Young et al. 1980; Laing \& Bridle 1987), which is a strong indication of an AGN. The flux of the core at $5 \mathrm{GHz}$ was measured to $S_{\nu}=160 \mathrm{mJy}$ (Nagar et al. 2002), giving $\log R_{\mathrm{X}}=-1.3$, which means that this source is radio-loud. The total X-ray luminosity in the central 2.5 radius region is $L_{2-10 \mathrm{keV}}=4.4 \times$ $10^{39} \mathrm{erg} \mathrm{s}^{-1}$ and the luminosity of the AGN is $L_{2-10 \mathrm{keV}}=4 \times 10^{39} \mathrm{erg} \mathrm{s}^{-1}$ (obtained by integrating the power-law spectral component). So the contribution from the LLAGN to the X-ray luminosity is $90 \%$.

NGC 4438 (L1, S0/a) has only one point source, which is close to the center but not exactly centered (within $2 \sigma$ ). It has a hard X-ray spectrum and is embedded in soft diffuse emission with an elongated morphology. The spectrum of the central source is well fitted by a thermal plasma model or a plasma component plus a power-law with a total luminosity of $L_{0.5-10 \mathrm{keV}} \sim 7.7 \times 10^{39} \mathrm{erg} \mathrm{s}^{-1}$. Since the source is deeply embedded in diffuse emission, it is possible that it is an AGN. This source is weakly detected at $1.49 \mathrm{GHz}$ with a flux of $116 \mathrm{mJy}$ (Condon 1987), which gives $\log R_{\mathrm{X}}<-1.2$, i.e. it can be radio loud. Also a broad $\mathrm{H} \alpha$ line was detected (Ho et al. 1997b), which supports the AGN hypothesis. An amorphous patch about 5"in size was detected in the UV (Maoz et al. 1996), which was interpreted as a superbubble and might be associated with the extended patch of diffuse emission seen in the X-ray. However, age estimates of the stellar population indicate that an old population dominates the optical starlight spectrum in the bulge (Cid Fernandes et al. 2005; Cid Fernandes et al. 2004; Bonatto et al. 2000). We estimated the X-ray luminosity from the central $2 . .5$ radius region to be $L_{2-10 \mathrm{keV}}=1.4 \times$ $10^{39} \mathrm{erg} \mathrm{s}^{-1}$ and the luminosity of the AGN to be $L_{2-10 \mathrm{keV}}=1.4 \times 10^{39} \mathrm{erg} \mathrm{s}^{-1}$ (obtained by integrating the power-law component). Thus the hard $\mathrm{X}$-ray luminosity of this LINER is primarily powered by the LLAGN. We note that this is the only LINER whose dominant X-ray source luminosity changes depending of what band we consider. The AGN dominates in the $2-10 \mathrm{keV}$ band, while the plasma emission dominates in the $0.5-10 \mathrm{keV}$ band.

NGC 4457 (L2, S0/a) has one bright central source with a hard spectrum, embedded in soft diffuse emission. The spectrum of the central source is well fitted by a power-law plus plasma model or a two-temperature plasma model $\left(L_{0.5-10 \mathrm{keV}}=1.9 \times 10^{39} \mathrm{erg} \mathrm{s}^{-1}\right)$. This can be interpreted as the spectrum of an embedded AGN and is confirmed by the detection of this source at $1.49 \mathrm{GHz}$ (Condon 1987). From this detection we infer $\log R_{\mathrm{X}}=-1.6$, which means that this source is radio loud. The luminosity of the AGN is $L_{2-10 \mathrm{keV}}=9.7 \times 10^{38} \mathrm{erg} \mathrm{s}^{-1}$ while the luminosity of the central $2 . .5$ is $L_{2-10 \mathrm{keV}}=1.8 \times 10^{39} \mathrm{erg}$ $\mathrm{s}^{-1}$ so the AGN contributes $\sim 55 \%$ of the X-ray luminosity. This rest of the luminosity comes from the diffuse emission, so this LINER is a composite.

NGC 4494 (L2, E1-2) has six point sources in its central kiloparsec and the coordinates of the brightest source agree with the center of the galaxy within 2 $\sigma$. This source has a hard X-ray spectrum, which is best fitted by a two-temperature plasma model or a power-law. The luminosity is $L_{0.5-10 \mathrm{keV}}=$ $1.6 \times 10^{39} \mathrm{erg} \mathrm{s}^{-1}$. There is no detection of a source at $5 \mathrm{GHz}$, with an upper limit of $0.5 \mathrm{mJy}$ (Wrobel \& Heeschen 1991), leading to $\log R_{\mathrm{X}}<$ -3.7 . Thus, this source could be radio loud and could be an AGN. The luminosity of the central $2^{\prime \prime} .5$ is $L_{2-10 \mathrm{keV}}=1.1 \times 10^{39} \mathrm{erg} \mathrm{s}^{-1}$ and the luminosity of the power AGN is $L_{2-10 \mathrm{keV}}=9.9 \times 10^{38} \mathrm{erg} \mathrm{s}^{-1}$ so the AGN candidate contributes $90 \%$ of the luminosity. The stellar population was estimated to be less than 5 Gyr old (O'Sullivan \& Ponman 2004).

NGC 4552 (T2, E1) has a bright, hard central source, plus four other point sources in its central kiloparsec and soft diffuse emission. The spectrum of the central source is well fitted by a power-law or a comptonized blackbody. These models give a luminosity of $L_{0.5-10 \mathrm{keV}}=3.7 \times 10^{39} \mathrm{erg} \mathrm{s}^{-1}$. This source was clearly detected at $4.85 \mathrm{GHz}$ with $S_{\nu}=64 \mathrm{mJy}$ and a spectral index $p=-0.23$ (Condon et al. 1991) so we calculate $\log R_{\mathrm{X}}=$ -1.5 , which means that this source is radio loud. Moreover a UV flare from this source was detected (Renzini et al. 1995; Cappellari et al. 1999) 
and the UV flux was observed to vary over a short period of time (Maoz et al. 2005) indicating a weak AGN. The stellar population was estimated to be old (9.6 Gyr, Terlevich \& Forbes 2002). The $\mathrm{X}$-ray luminosity from the central $2 . .5$ region is $L_{2-10 \mathrm{keV}}=3.4 \times 10^{39} \mathrm{erg} \mathrm{s}^{-1}$ and the luminosity of the AGN is $L_{2-10 \mathrm{keV}}=2.0 \times 10^{39} \mathrm{erg} \mathrm{s}^{-1}$. Thus the X-ray luminosity of this LINER comes mostly from its $\mathrm{AGN}(\sim 60 \%)$.

NGC 4636 (L1, E1/S0) has bright knotty, soft, diffuse emission. There are five point sources deeply embedded in the diffuse emission. One of them is at the center but this is not the brightest source and its spectrum is soft. This source did not have enough counts to justify fitting its spectrum with a variety of models, but its luminosity was evaluated to be $L_{0.5-10 \mathrm{keV}}=8.7 \times 10^{38} \mathrm{erg} \mathrm{s}^{-1}$. A broad $\mathrm{H} \alpha$ component was detected (Ho et al. 1997b) indicating a LLAGN, but the X-ray signature of an AGN is not detected. Loewenstein et al. (2001) failed to detect an AGN using the same data set, as did Terashima et al. (2002) using ASCA observations. If there is an AGN (as suggested by the detection of a broad Holine ), it could be heavily absorbed.

NGC 5055 (T2, Sb/c) has a five point sources in its nucleus, clustered close to the center, and a bright hard source at the very center. The spectrum of this source is well fitted by a two-temperature plasma model, but a power-law plus plasma model gives a very poor fit. We did not attempt more complex models due to the small number of degrees of freedom. Maoz et al. (1995) resolved the central point source in the UV and obtained a radius of $7 \mathrm{pc}$ (at the distance assumed in this paper). Maoz et al. (2005) did not observe any variation of the UV flux of this source. All these pieces of evidence indicate that this source is more likely a compact star cluster. This source is not detected at various radio frequencies except at $57.5 \mathrm{MHz}$ with $S_{\nu}=2.1$ Jy (Israel \& Mahoney 1990), which means that this source is radio quiet $\left(R_{X}=-6\right)$ and not likely to be an AGN. The stellar population of NGC 5055 has a range of ages from $1 \mathrm{Myr}$ to 10 Gyr with most of the population of intermediate and old age (Gonzalez Delgado et al. 2004; Cid Fernandes et al. 2004). So the X-ray emission of this LINER is powered by stellar processes.

NGC 5866 (T2, S0) has a central, hard X-ray source and one other point source far away from the center and embedded in soft diffuse emission. The spectrum of the central source did not have enough counts to attempt a fit. However its luminosity was estimated to be $L_{0.5-10 \mathrm{keV}}=3.2 \times 10^{38} \mathrm{erg} \mathrm{s}^{-1}$ with $98 \%$ of it emitted in the hard band. This is also the luminosity from the central 2.5 " region indicating that this source is the main contributor to the central X-ray luminosity. This source has $S_{\nu}=7.5$ mJy at $5 \mathrm{GHz}$ (Nagar et al. 2000). We calculated that $\log R_{\mathrm{X}}=-1.5$, which means that this source is radio loud and possibly a LLAGN.
NGC 7331 (T2, Sb) has three point sources but none are at the center (at the $3 \sigma$ confidence level). There is no hard X-ray emission close to the center of the galaxy. No source had enough counts to justify spectral fits. The stellar population does not have a young component and is mostly 1-10 Gyr old (Cid Fernandes et al. 2005; Cid Fernandes et al. 2004; Gonzalez Delgado et al. 2004). So this LINER might be powered by stellar processes, most likely associated with the old stellar population.

\section{SUMMARY OF RESULTS AND DISCUSSION}

\subsection{Summary and Immediate Implications for $A G N$ Demographics}

In order to generalize our results, the first issue we examine is whether the present sample is representative of the entire population of LINERs, in terms of the distribution of LINER classes. The ratio of transition objects to pure LINERs in the Ho et al. (1997c) survey is 0.75 while in our sample, it is 0.63 (ignoring NGC 1553, which was not classified by Ho). So the transition objects are slightly underrepresented in our sample. Moreover in our sample, $27 \%$ of the pure LINERs are L1, which is comparable to the $25 \%$ of L1 LINERs in the Ho et al. (1997c) survey. We also note that the $\mathrm{H} \alpha$ luminosity distribution of the sample closely resembles that from the Ho et al (1997c) sample. Thus, one can generalize the results from this sample with reasonable confidence.

Soft, circumnuclear diffuse emission is common in our objects with a typical $0.5-2 \mathrm{keV}$ luminosity on the order of $10^{38} \mathrm{erg} \mathrm{s}^{-2}$, in agreement with the results of GonzálezMartin et al. (2005, 2006). In 12 out of 19 LINERs there are signs of an AGN whether it dominated the X-ray luminosity or not. In 5/19 LINERs we did not detect an AGN, with upper limits to the luminosity as listed in Table 4. For 2/19 LINERs (NGC 4636 and NGC 7331), it was difficult to determine whether there was an AGN because of the knotty structure of the diffuse emission. Thus, at least $63 \%$ (and possibly up to $74 \%$ ) of the objects in the sample harbor an AGN.

Table 4 summarizes the $0.5-2$ and $2-10 \mathrm{keV}$ luminosity of the AGN candidates in each galaxy (Columns 6 and 7) along with their Eddington ratio (Column 8; see details below) and X-ray radio loudness parameter (Column 9). When comparing the $2-10 \mathrm{keV}$ luminosity of the AGN (Column 4) to the luminosity of the diffuse emission (Columns 2 and 3), we notice that for 10 of the 12 galaxies with an AGN candidate, the AGN dominates the $2-10 \mathrm{keV}$ luminosity in the inner 2 . 5 ; in 9 out of these 10 cases, the AGN dominates the total 2-10 keV luminosity in the inner kiloparsec. When we consider the luminosity in the entire $0.5-10 \mathrm{keV}$ band, however, the contribution of the diffuse emission is often comparable to the contribution of the AGN, even in the inner 2". 5 . Figure 5 shows the distribution of luminosities of the AGNs found in our sample (and upper limits when there is no detection of an AGN), the distribution of $\log R_{\mathrm{X}}$ and the distribution of the fractional contribution of the AGN to the $0.5-10 \mathrm{keV}$ luminosity of the central 2.5 region. We note that the detected AGNs span a wide range in $2-10 \mathrm{keV}$ luminosity, from $1.7 \times 10^{37}$ to $1.2 \times 10^{40} \mathrm{erg} \mathrm{s}^{-1}$, with an average of 
$L_{2-10 \mathrm{keV}}=2.3 \times 10^{38} \mathrm{erg} \mathrm{s}^{-1}$. This luminosity range is comparable to that found by Terashima \& Wilson (2003) for LLAGN in LINERs with bright, compact radio sources.

We can express the Eddington ratio of the AGNs we have detected (the ratio of the bolometric luminosity of the AGN and the Eddington luminosity) as follows:

$$
\begin{gathered}
\mathcal{R}_{\mathrm{Edd}} \equiv \frac{L_{\mathrm{bol}}}{L_{\mathrm{Edd}}}=\frac{L_{2-10 \mathrm{keV}} / \zeta}{1.3 \times 10^{38}\left(M_{\mathrm{BH}} / \mathrm{M}_{\odot}\right) \mathrm{erg} \mathrm{s}^{-1}} \\
=7 \times 10^{-7} L_{38} \zeta_{-1}^{-1} M_{7}^{-1},
\end{gathered}
$$

where $M_{B H}=10^{7} M_{7} \mathrm{M}_{\odot}$ is the black hole mass reported in Table $1, L_{2-10 \mathrm{keV}}=10^{38} L_{38} \mathrm{erg} \mathrm{s}^{-1}$ is the X-ray luminosity (reported in Table 4 ) and $\zeta=0.1 \zeta_{-1}$ is a "bolometric correction" (see Ho 1999). The resulting values of $\mathcal{R}_{\mathrm{Edd}}$ are included in column 8 of Table 4; they span the range $-8.2<\log \mathcal{R}_{\text {Edd }}<-5.1$, for detected AGNs in our sample. These values are comparable to those found by Filho et al. (2004), for a sample of transition-type LINERs (using the same method for black hole mass determination). These results however are significantly different from those of Satyapal et al. (2005) who find $-6.7<\log \mathcal{R}_{\text {Edd }}<-0.3$ and $2 \times 10^{38}<L_{2-10 \mathrm{keV}}<10^{42} \mathrm{ergs}^{-1}$ for the candidate AGNs in their infrared-bright LINER sample. We note that they used a bolometric correction three times higher than the one we use, but this does not account for the whole difference. Their AGNs have higher X-ray luminosities so the very low Eddington ratios AGNs in our sample are probably the very low luminosity AGNs missing from their sample.

\subsection{Comparison With Normal Galaxies and the Source of Fuel for the AGN}

The X-ray luminosities and Eddington ratios of the accreting black holes in our sample of LINERs are very similar to those of black holes in the "quiescent" galaxies studied by Soria et al. (2006a,b). Moreover, the properties of the diffuse emission (gas temperature, density, and soft Xray luminosity; see §3.3) as well as the black hole masses in the two samples are quite similar. Therefore, we can infer that the Bondi accretion rates should also be similar. Indeed, an inspection of Figure 5 of Soria et al. (2006b) shows that several of the LINERs in our sample fall in the same region of the Eddington ratio vs Bondi rate diagram. As Soria et al. (2006b) demonstrate, the fuel necessary to power the observed low-level activity in their sample can easily be provided by a combination of mass loss from stars within the black hole's "sphere of influence" and from the flow of hot interstellar gas into that sphere from larger radii (Bondi accretion). In fact, the hot interstellar gas itself is likely to have originated in stellar mass loss over a larger volume around the center of the galaxy (see our earlier discussion in §3.3). The same interpretation applies to the LINERs that we study here, based on the close similarities between the nuclear properties of the two samples.

These similarities also contribute to the puzzle of why some galaxies host LINERs while others do not. To address this question we examine the energy budget of the AGN in the next section in the broader context of the power-source behind the optical emission-line spectra of LINERs.

\subsection{The Role of the AGN in the Grand Scheme of Things}

In this sample, stellar processes, as manifested by soft, diffuse emission, seem to frequently contribute to the energy budget of the central 2 '. 5 region, whether alone or in combination with an AGN. The AGN is often not the major power source. To illustrate this we plot in Figure 6 the luminosity from the diffuse emission vs the luminosity from the AGN in the $0.5-10 \mathrm{keV}$ and $2-10 \mathrm{keV}$ bands. In more than half of the cases the diffuse emission dominates. It would be interesting to determine whether the dominant contribution to the X-ray luminosity depends on the LINER classification or the Hubble type, but our sample is too small to allow any firm conclusions on these questions.

To investigate the role of the AGN in powering the observed optical emission lines, we looked for correlations between the different X-ray luminosities and the $\mathrm{H} \alpha$ luminosity (taken from Ho et al 1997a). We find a correlation between $L_{\mathrm{H} \alpha}$ and $L_{2-10 \mathrm{keV}}$ of the central 2.55 region (Spearman rank correlation coefficient $r_{\mathrm{s}}=0.33$ ). Our objects fall in the same region of the $L_{2-10 \mathrm{keV}^{-}} L_{\mathrm{H} \alpha}$ diagram as the lowest-luminosity objects of Ho et al. (2001) and also very close to the correlation between these two quantities followed by low-redshift quasars and Seyfert 1 galaxies. This can be seen in Figure 7 where the squares represent the data from our sample and the stars represent the LINERs observed by Ho et al. $(2001)^{8}$. We also draw the best fit found by Ho et al. (2001) as the solid line and the range of luminosity where a starburst would fall (dotted lines). The latter locus is obtained by combining relations between these two quantities and the star formation rate (Kennicutt 1998; Ranalli, Comastri, \& Cetti 2003; Grimm, Gilfanov, \& Sunyaev 2003; Colbert et al. 2004; Persic et al. 2004). It is noteworthy that none of our objects are close to the starburst locus, which is consistent with an absence of young stellar populations in the majority of the galaxies in our sample.

In Figure 8, we focus on the correlation between the 2..5 X-ray and $\mathrm{H} \alpha$ luminosities for the objects in our sample. The trend persists regardless of whether we use the $2-10 \mathrm{keV}$ or the $0.5-2 \mathrm{keV}$ luminosity. In fact, the correlation appears stronger when considering the soft X-ray band instead of the hard X-ray band: the Spearman rank correlation coefficients are 0.48 and 0.33 in the soft and hard bands respectively. This suggests that production mechanism of the $\mathrm{H} \alpha$ line is not strongly correlated to the source of the hard X-ray radiation.

Figure 8 gives a preliminary indication that photoionization by the AGN is unlikely to be responsible for powering the optical emission lines. We have carried out the following tests to investigate this issue further.

1. The first test is based on energy balance between the emission lines and the ionizing continuum. Assuming that the ionizing continuum is a power-law

${ }^{8}$ Ho et al. (2001) scaled a power-law model with $\Gamma=1.8$ and $N_{\mathrm{H}}=2 \times 10^{20} \mathrm{~cm}^{-2}$ to the observed count rate of the central sources to estimate the luminosity of the sources. The objects from Ho et al. (2001) that do not follow the correlation are mostly transition-type LINERs, which were not included in the calculation of the correlation. 
of photon index $\Gamma$ from 1 Ry to $100 \mathrm{keV}$ (consistent with ADAF models), its total luminosity can be obtained from the observed $2-10 \mathrm{keV}$ luminosity via $L_{i}=I(\Gamma) L_{2-10 \mathrm{keV}}$, where the function $I(\Gamma)$ has values between 5 and 12 for $\Gamma$ in the range 1.1-1.9. Thus for the ionizing continuum to power the $\mathrm{H} \alpha \mathrm{lu}$ minosity alone, we require that $L_{2-10 \mathrm{keV}}>5 L_{\mathrm{H} \alpha}$. Out of the 19 objects in our sample, only four satisfy this condition, under the most optimistic assumption. In the case of NGC 1553, NGC 4374 NGC 4494, and NGC 4552 the ionizing continuum luminosity exceeds the $\mathrm{H} \alpha$ luminosity by a factor of a few in the best case. Additional power would be available, if the AGN SED included a significant "UV bump", but this appears rather unlikely, in view of the LLAGN SED shapes shown by Ho (1999). It is also unlikely that we have underestimated the X-ray luminosity of the AGN because of absorption. This is because the spectra of $2 / 3$ of our AGN have a sufficiently high $\mathrm{S} / \mathrm{N}$ that we were able to fit them, determine the absorbing column, and correct the luminosity (of course, we cannot rule out the possibility that the entire X-ray emission is scattered and that we do not have a direct view of the AGN).

2. Another test for the photoionization hypothesis relies on photon counting. Assuming the same continuum shape as above, we can express the ionizing photon rate produced by the AGN in terms of the observed X-ray luminosity as $Q_{i}=6.25 \times$ $10^{47} G(\Gamma)\left(L_{2-10 \mathrm{keV}} / 10^{39} \mathrm{erg} \mathrm{s}^{-1}\right) \mathrm{s}^{-1}$. The function $G(\Gamma)$ has values between 2 and 30 for $\Gamma$ in the range 1.1-1.9. By relating this to the $\mathrm{H} \alpha$ photon rate, we can derive the following requirement for the $\mathrm{H} \alpha$ luminosity: $L_{\mathrm{H} \alpha}<0.007 f G(\Gamma) L_{2-10} \mathrm{keV}$, where $f$ is the fraction of ionizing photons absorbed by the emission-line gas and one in 2.2 recombinations leads to the emission of an $\mathrm{H} \alpha$ photon (Osterbrock 1989; we have assumed that there are no secondary ionizations or excitations by fast photoelectrons). None of the objects in our sample satisfy this condition, even if $f=1$. The four objects identified in the previous paragraph could satisfy this condition if each ionizing photon leads to $2-5$ ionizations, on average. All other objects would require more than 10 secondary ionizations; most require more than 100 .

Thus the AGNs in the objects of our present sample are not energetically significant. It is also noteworthy that in more than half the cases, the electromagnetic power of the AGN is not enough to drive the diffuse X-ray emission. Examining the samples of Terashima \& Wilson (2003) and Filho et al. (2004), we notice a similar effect. In particular, the former sample includes AGNs typically an order of magnitude more luminous than ours but the faintest $25 \%$ of their AGNs fail the energy budget test and cannot power the $\mathrm{H} \alpha$ emission. In the latter sample the X-ray detected AGNs have comparable luminosities to ours and they all fail the energy budget test. ${ }^{9}$ It appears, at least at first glance, that as the luminosity of the AGN decreases, its important as the power source of the optical emission lines diminishes too. Therefore, we conclude our discussion by considering other possible sources of power for the optical emission lines below.

\subsection{Epilogue: What Is the Power Source of the Optical Emission Lines?}

The analysis presented above shows that the properties of the LINERs in the present sample are very similar to those of normal galaxies. More specifically, the hot interstellar gas in these LINERs has, on average, the same temperature, density, and luminosity as in normal galaxies. Moreover, the average properties of the stellar populations in these LINERs are similar to those of normal galaxies: the X-ray binary luminosity functions are the same and in most of our LINERs the optical spectra indicate old stellar populations. Finally, even though an AGN is often detected, its electromagnetic power does not appear to be significant. Thus we must look for an explanation for the LINER optical spectra that involves either stellar processes or a mechanical interaction of the AGN with its immediate environs.

In three of the LINERs in our sample (NGC 3507, NGC 3628, and NGC 5055) there is evidence for a young stellar population, while in one other object (NGC 2681) there is evidence for an intermediate-age population. In more general terms, Cid Fernandes et al. (2005) find that $38 \%$ of LINERs and transition objects have intermediateage stellar populations in their very nuclei. Hot stars from a young stellar population are obvious sources of ionizing photons that could power the optical emission lines (see \$1). In old and especially in intermediate-age stellar populations, ionizing photons can be provided by post-AGB stars and the central stars of planetary nebulae (Binette et al. 1994).

An alternative explanation is that the AGN powers the emission lines by depositing mechanical energy via outflows (jets or winds) into the emission line gas. At the low Eddington ratios that we find here, the kinetic power of jets from the AGN can be significantly higher (by up to 3 orders of magnitude) than the electromagnetic luminosity (see Nagar, Falcke, \& Wilson 2005). Some of this power may be dissipated by shocks in the immediate vicinity of the AGN, which would naturally lead to LINER-like emission line spectra. This hypothesis is bolstered by the observation that the emission-line regions of LINERs are rather amorphous or filamentary on scales of $10-100 \mathrm{pc}$ (Pogge et al. 2000). This type of feedback from the AGN may also contribute to additional heating of the diffuse gas, which in turn would limit the accretion rate and lead to the very low Eddington ratio that we infer (see the discussion by Soria et al. 2006b).

In conclusion, we note that even though some LINERs are obviously powered by their AGNs (M81 and NGC 4579 are among the best known examples), the electromagnetic luminosity of the AGN is not always enough to account for

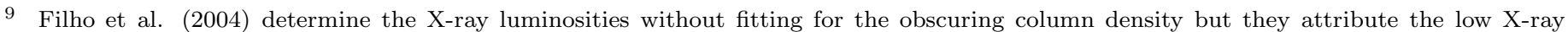
luminosity to absorption. Their three longest observations are analyzed here as well. In NGC 4552 we find that intrinsic luminosity of the AGN, after correcting for absorption, is not enough to balance the energy budget. In NGC 5866 there are too few counts to warrant a fit, and in NGC 7331, we do not detect an AGN at all. 
the observed emission-line luminosity. All of the physical processes discussed in the literature so far may contribute to powering the emission line spectra, making LINERs a heterogeneous class of objects and suggesting that AGN photoionization may be the exception rather than the rule.

This work was supported by the National Aeronautics and Space Administration through Chandra award num- ber AR4-5010A issued by the Chandra X-Ray Observatory Center, which is operated by the Smithsonian Astrophysical Observatory for and on behalf of the National Aeronautics and Space Administration under contract NAS803060. We would like to thank Patrick Broos and Leisa Townsley for their assistance with the use of the ACIS Extract software. We also thank the anonymous referee for helpful comments.

\section{REFERENCES}

Argyle, R.W., Clements, E.D. 1990, Obs, 110, 93

Arnaud, K.A. 1996, in ASP Conf. Ser. 101, Astronomical Data Analysis Software and Systems V, ed. Jacoby, G. \& Barbes J. (San Francisco:ASP) 17

Barret, D. 2001, AdSpR, 28, 307

Barth, A.J., Filippenko, A.V., \& Moran, E.C. 1999, ApJ, 525, 673

Barth, A.J., Ho, L.C., Filippenko, A.V., Rix, H.-W., \& Sargent, W.L.W. 2001, ApJ, 546, 205

Barth, A.J., \& Shields, J.C. 2000, PASP, 112, 753

Barth, A. J., Ho, L. C. \& Sargent, W. L. W. 2002, AJ, 124, 2607

Benedict, G.F., Howell, D.A., Jørgensen, I., Kenney, J.D.P., \& Smith, B.J. 2002, AJ, 123, 1411

Binette, L., Magris, C.G., Stasinska, G., \& Bruzual, A.G. 1994, A\&A, 292, 13

Blandford, R.D., \& Begelma, M.C. 1999, MNRAS, 303, 1

Blanton, E.L., Sarazin, C.L., Irwin, J.A. 2001, ApJ, 552, 106

Boker, T., 2000, in ASP Conf. Ser. 211, Massive Stellar Clusters, ed. A. Lancon \& C.M. Boily (San Francisco:ASP), 227

Bonatto, C., Bica, E., Pastoriza, M.G., \& Alloin, D, 2000, A\&A, 355, 99

Borozdin, K., Revnivtsev, M., Trudolyubov, S., Shrader, C., \& Titarchuk, L. 1999, ApJ, 517, 367

Botte, V., Ciroi, D., di Mille, F., Rafanelli, P., \& Romano, A. 2005, MNRAS, 356, 789

Bower, G.A., Wilson, A.S., Heckman, T.M., \& Richstone, D.O. 1996, AJ, 111,1901

Broos, P.S., Townsley, L.K., Getman, K., \& Bauer, F.E. 2002, ACIS Extract, An ACIS Point Source Extraction Package (University Park: Pennsylvania State Univ.)

Burstein, D., Bertola, F., Buson, L.M., Faber, S.M., \& Lauer, T.R. 1988, ApJ, 328, 440

Cappellari, M., Bertola, F., Burstein, D., Buson, L.M., Greggio, L., \& Renzini, A. 2001, ApJ, 551, 197

Cappellari, M., Renzini, A., Greggio, L., di Serego Alighieri, S., Buson, L.M., Burstein, D.,\& Bertola, F. 1999, ApJ, 519, 117

Carollo, M. 1999, ApJ, 523, 566

Colbert, E.J.M., Heckman, T.M., Ptak, A.F., Strickland, D.K., \& Weaver, K.A. 2004, ApJ, 602, 231

Combes, F. 2001 Advanced Lectures on the Starburst-AGN Connection, eds, I. Aretxaga, D. Kunth, \& R. Mújica (Singapore:: World Scientific), 223

Condon, J.J. 1987, ApJSS, 65, 485

Condon, J.J., Frayer, D.T., \& Broderick, J.J. 1991, AJ, 101, 362

David, L. P., Jones, C., Forman, W., \& Murray, S. S. 2005, ApJ, 635, 1053

De Young, D.S., Butcher, H., \& Condon, J.J. 1980, ApJ, 242, 511

Dickey, J.M., \& Lockman, F.J. 1990, ARA\&A, 28, 215

Dopita, M.A, Koratkar, A.P., Evans, I.N., Allen, M., Bicknell, G.V., Sutherland, R.S., Hawley, J.F., \& Sadler, E. 1996, in The physics of Liners in view of recent observations. ASP Conference Series, Vol. 103, ed. Eracleous, M., Koratkar, A., Leitherer, C., \& Ho, L., p.44

Dopita, A.M., \& Sutherland, R.S. 1995, ApJ, 455, 468

Eracleous, M., \& Halpern, J.P. 2001, 554, 240

Ferland, G.J., \& Netzer, H. 1983, ApJ, 264, 105

Cid Fernandes, R.C., Gonzalez Delgado, R.M., Storchi-Bergmann, T., Pires Martins L., \& Schmitt, H. 1005, MNRAS, 356, 270

Cid Fernandes, R.C., et al. 2004, ApJ, 605, 105

Eracleous, M., Shields, J.C., Chartas, G., \& Moran, E.C. 2002, ApJ, 565,108

Ferrarese, L. \& Merrit, D. 2000, ApJ, 539, L9

Fey, A.L., Ma, C., Arias, E.F., Charlot, P., Feissel-Vernier, M., Gontier, A.-M., Jacobs, C.S., Li, J., \& MacMillan, D.S. 2004, AJ, 127,3587

Filippenko, A.V. 1996, in ASP Conf. Ser. 103, The physics of Liners in view of recent observations, ed. Eracleous, M., Koratkar, A., Leitherer, C., \& Ho, L. (San Francisco:ASP) 17

Filippenko, A.V., \& Halpern, J.P. 1984, ApJ, 285, 458

Filippenko, A.V., \& Terlevich, R. 1992, ApJ, 397, L79

Filho, M.E., Fraternali, F., Markoff, S., Nagar, N.M., Barthel, P.D., Ho, L.C., \& Yuan, F. 2004, A\&A, 418, 429
Forman, W., Jones, C., \& Tucker, W. 1985, ApJ, 293, 102

Freeman, P.E. et al. 2002, ApJSS, 138,185

Garmire, G.P., Bautz, F.W., Ford, P.G., Nousek, J.A., \& Ricker, G.R. 2003, Proc. SPIE, 4851, 28

Gebhardt, K., et al. 2000, ApJ, 539, L13

Gonzalez Delgado, R.M., Cid Fernandes, R., Perez, E., Martins, L.P., Storchi-Bergmann, T., Schmitt, H., Heckman, T., \& Leitherer, C. 2004

González-Martin, O., Masegosa, J., Márquez, J., Dultzin-Hacyan, D., \& Guerrero, M. A. 2005, Mem.S.A.It., 76, 91

González-Martin, O., Masegosa, J., Márquez, J., Dultzin-Hacyan, D., \& Guerrero, M. A. 2005, in Populations of High Energy Sources in Galaxies (proc. IAU Symp. 230), in press

Gregg, M.D. 1989, ApJ, 337, 45

Gregg, M.D., Ferguson, H.C., Minniti, D., Tanvir, N., \& Catchpole, R. 2004, AJ, 127, 1441

Grimm, H.-J., Gilfanov, M., \& Sunyaev, R. 2002, MNRAS, 339, 793

Halderson, E.L., Moran, E.C., Filippenko, A.V., \& Ho, L.C. 2001, ApJ, 122, 637

Halpern, J.P., \& Steiner, J.E. 1983, ApJ, 269, L37

Harnett, J.I. 1987, MNRAS, 227, 887

Heckman, T.M 1980, A\&A, 87, 152

Ho, L.C. 1996, in ASP Conf. Ser. 103, The physics of Liners in view of recent observations, ed. Eracleous, M., Koratkar, A., Leitherer, C., \& Ho, L. (San Francisco:ASP) 70

Ho, L.C. 1999, ApJ, 516, 672

Ho, L.C., Feigelson, E.D., Townsley, L.K., Sambruna, R.M., Garminre, G.P., Brandt, W.N., Filippenko, A.V., Griffiths, R.E., Ptak, A.F. \& Sargent, W.L.W. 2001, ApJ, 549, L51

Ho, L.C., Filippenko, A.V., \& Sargent, W.L.W. 1997a, ApJS, 112, 315

Ho, L.C., Filippenko, A.V., Sargent, W.L.W., \& Peng, C. 1997b, ApJS, 112, 391

Ho, L.C., Filippenko, A.V., \& Sargent, W.L.W. 1997c, ApJ, 487, 568

Ho, L.C, Rudnick, G., Rix, H.-W., Shields, J.C., McIntosh, D.H., Filippenko, A.V., Sargent, W.L.W., \& Eracleous, M. 2000, ApJ, 541,120

Hummel, E., \& Saikia, D.J. 1991, A\&A, 249, 43

Immler, S., Wang, Q.D., Leonard, D.C., \& Schlegel, E.M. 2003, ApJ, 595,727

Israel, F.P., \& Mahoney, M.J. 1990, ApJ, 352, 30

Kaastra, J.S. 1992, An X-Ray Spectral Code for Optically Thin Plasmas (Internal SRON-Leiden Report, updated version 2.0)

Laing, R.A., \& Bridel, A.H. 1987, MNRAS, 228, 557

Kennicutt, R. C. 1998, ARA\&A, 36, 189

Lasota, J.P., et al. 1996, ApJ, 462, 142

Laurent, P., \& Titarchuk, L. 1999, ApJ, 511, 289

Liedahl, D.A., Osterheld, A.L., and Goldstein, W.H. 1995, ApJL, 438,115

Loewenstein, M., Mushotzky, R.F., Angelini, L., \& Arnaud, K.A. 2001, ApJ, 555, L21

Longhetti, M., Bressan, A., Chiosi, C., \& Rampazzo, R. 2000,A\&A, 353,917

Makishima, K., Maejima, Y., Mitsuda, K., Bradt, H.,V., Remillard, R.A., Tuohy, I.R. Hoshi, R. \& Nakagawa, M. 1986, ApJ 308, 635

Maoz, D., Filippenko, A.V., Ho, L.C., Rix, H.-W, Bahcall, J.N., Schneider, D.P., \& Macchetto, F.D. 1995, ApJ, 440, 91

Maoz, D., Filippenko, A.V., Ho, L.C., Macchetto, F.D., Rix, H.-W., \& Schneider, D.P. 1996, ApJS, 107, 215

Maoz, D., Nagar, N.M., Falcke, H., \& Wilson, A.S. 2005, ApJ, 625,699

Mewe, R., Gronenschild, E.H.B.M., and van den Oord, G.H.J. 1985, A\&AS, 62, 197

Mewe, R., Lemen, J.R., and van den Oord, G.H.J. 1986, A\&AS, 65, 511

Mitsuda, K., Koyama, K., Makishima, K., Matsuoka, M., Ogawara, Y., Suzuki, K., Tanaka, Y., Shibazaki, N., \& Hirano, T. 1984, PASJ, 36, 741

Moran, E.C., Halpern, J.P., \& Helfand, D.J 1996, ApJS, 106, 341

Morrison, R., \& McCammon, D. 1983, ApJ, 270, 119

Mushotzky, R.F., Done, C., \& Pounds, K. A. 1993, ARA\&A, 31, 717 
Nagar, N.M., Falcke, H., \& Wilson, A.S. 2005, A\&A, 435, 521

Nagar, N.M., Falcke, H., Wilson, A.S., \& Ho, L.C. 2000, ApJ, 542, 186

Nagar, N.M., Falcke, H., Wilson, A.S., \& Uvelstad, J.S. 2002, A\&A, 392,53

Narayan, R., \& Yi, I. 1994, ApJ, 428, L13

Narayan, R., \& Yi, I. 1995, ApJ, 444, 231

Nishimura, J., Mitsuda, K., \& Itoh, M. 1986, PASJ, 38, 819

Osterbrock, D. E. 1989, Astrophysics of Gaseous Nebulae and active Galactic Nuclei (Mill Valley: University Science Books)

O'Sullivan, E., \& Ponman, T.J., 2004, MNRAS, 349, 535

Persic, M., \& Rephaeli, Y. 2001, A\&A, 382, 843

Persic, M., Rephaeli, Y., Braito, V., Cappi, M., Della Ceca, R.,Franceschini, A., \& Gruber, D. E. 2004, A\&A, 419, 849

Phillips, M.M., Jenkins, C.R., Dopita, M.A., Sadler, E.M., \& Binette, L. 1986, AJ, 92, 1062

Pogge, R.W., Maoz, D., Ho, L.C., \& Eracleous, M. 2000, ApJ, 532, 323.

Proctor, R.N., \& Sansom, A.E. 2002, MNRAS, 333,517

Quataert, E., \& Narayan, R. 1999, ApJ, 516, 399

Ranalli, P., Comastri, A., \& Setti, G. 2003, å, 399, 39

Randall, S.W., Sarazin, C.L., \& Irwin, J.A. 2004, ApJ, 600, 729

Renzini, A., Greggio, L., di Serego-Alighieri, S., Cappellari, M., Burnstein, D., \& Bertola, F. 1995, Nature, 378,39

Sarazin, C.L., Irwin, J.A., \& Bregman, J.N. 2001, ApJ, 556,533

Schrader, C., \& Titarchuk, L. 1999, ApJ 521, L21

Shields, J.C. 1992, ApJ, 399, L27
Shields, J.C., Rix, H.-W., McIntosh, D.H., Ho, L.C., Rudnick, G., Filippenko, A.V., Sargent, W.L.W., \& Sarzi, M. 2000, ApJ, 543, L27

Sivakoff, G.R., Sarazin, C.L., \& Irwin, J.A. 2003, ApJ, 599, 218

Sivakoff, G.R., et al. 2004, ApJ, 617, 262

Soria, R., et al. 2006a, ApJ, in press (astro-ph/0511293)

Soria, R., et al. 2006b, ApJ, in press (astro-ph/051134)

Storchi-Bergmann, T., Baldwin, J.A., \& Wilson, A.S. 1993, ApJ, 410, L11

Strickland, D.K., Colbert, E.J.M., Heckman, T.M., Weaver, K.A., Dahlem, M., \& Stevens, I.R. 2001, ApJ, 560, 707

Terashima, Y., Iyomoto, N., Ho, L.C., \& Ptak, A.F. 2002, ApJS, 139, L36

Terashima, Y. \& Wilson, A.S. 2003, ApJ, 583, 145

Terlevich, A.I., \& Forbes, D.A. 2002, MNRAS, 330, 547

Terlevich, R., \& Melnick, J. 1985, MNRAS, 213, 841

Titarchuk, L., Mastichiadis, A., \& Kylafis, N.D. 1997, ApJ, 487, 834

Titarchuk, L., \& Zannias, T. 1998, ApJ,493, 863

Tremaine, S. et al. 2002, ApJ, 574, 740

Trinchieri, G., Fabbiano, G., \& Canizares, C.R. 1986, ApJ, 310, 637

Veron-Cetty, M.-P. \& Veron, P.1986, A\&AS, 66, 335

Veron, P., Veron, M.P., Bergeron, J., \& Zuidervijk, E.J. 1981, A\&A, 97,71

Wang, Q.D., Immler, S., Walterbos, R., Lauroesch, J.T., \& Breitschwerdt, D. 2001, ApJL, 555, 99

Wang, Q.D., Xhaves, T., \& Irwin, J.A. 2003, ApJ, 598, 969

Wrobel, J.M. \& Heeschen, D.S. 1991, AJ, 101, 148 
TABLE 1

Target Properties and Observation Details

\begin{tabular}{|c|c|c|c|c|c|c|c|c|}
\hline $\begin{array}{l}\text { Galaxy }^{\mathrm{a}} \\
(\mathrm{NGC})\end{array}$ & $\begin{array}{r}\text { LINER } \\
\text { Type }^{\mathrm{b}} \\
\end{array}$ & $\begin{array}{r}\text { Hubble } \\
\text { Type }^{\mathrm{b}}\end{array}$ & $\begin{array}{l}\text { Dist. }^{\mathrm{b}} \\
(\mathrm{Mpc})\end{array}$ & $\begin{array}{c}N_{\mathrm{H}}^{\mathrm{c}} \\
\left(10^{20} \mathrm{~cm}^{-2}\right)\end{array}$ & $\begin{array}{c}L_{\mathrm{H} \alpha}{ }^{\mathrm{d}} \\
\left(\operatorname{erg~s}^{-1}\right) \\
\end{array}$ & $\log \left(\frac{M_{\mathrm{BH}}}{\mathrm{M}_{\odot}}\right)^{\mathrm{e}}$ & $\begin{array}{c}\text { Exposure } \\
(\mathrm{ks})\end{array}$ & $\begin{array}{c}\text { Observation } \\
\text { Date }\end{array}$ \\
\hline 1553 & $\mathrm{~L} 2 / \mathrm{T} 2^{\mathrm{f}}$ & S0 & 16.1 & 1.50 & $6.61 \times 10^{38}$ & 8.0 & 34.17 & $2000 / 01 / 02$ \\
\hline 2681 & L1 & $\mathrm{SB} 0 / \mathrm{a}$ & 13.3 & 2.42 & $6.76 \times 10^{38}$ & 7.2 & 80.00 & $2001 / 01 / 30$ \\
\hline 3379 & $\mathrm{~T} 2$ & E1 & 8.1 & 2.75 & $8.71 \times 10^{37}$ & 8.3 & 31.92 & $2001 / 02 / 13$ \\
\hline 3507 & L2 & $\mathrm{SBb}$ & 19.8 & 1.63 & $2.45 \times 10^{39}$ & $\ldots$ & 39.76 & $2002 / 03 / 08$ \\
\hline 3607 & L2 & S0 & 19.9 & 1.48 & $8.51 \times 10^{38}$ & 8.4 & 39.00 & $2001 / 06 / 12$ \\
\hline 3608 & L2 & $\mathrm{E} 2$ & 23.4 & 1.49 & $1.91 \times 10^{38}$ & 8.1 & 39.00 & $2001 / 06 / 12$ \\
\hline 3628 & $\mathrm{~T} 2$ & $\mathrm{Sb}$ & 7.7 & 2.23 & $7.41 \times 10^{36}$ & 7.9 & 58.70 & $2000 / 12 / 02$ \\
\hline 4111 & L2 & So & 17.0 & 1.40 & $2.51 \times 10^{39}$ & 7.8 & 15.00 & $2001 / 04 / 03$ \\
\hline 4125 & $\mathrm{~T} 2$ & E6 & 24.2 & 1.84 & $9.12 \times 10^{38}$ & 8.4 & 65.08 & $2001 / 09 / 09$ \\
\hline 4314 & L2 & $\mathrm{SBa}$ & 9.7 & 1.78 & $2.82 \times 10^{38}$ & 7.2 & 16.28 & $2001 / 04 / 02$ \\
\hline 4374 & L2 & E1 & 16.8 & 2.60 & $7.76 \times 10^{38}$ & 8.8 & 28.85 & $2000 / 05 / 19$ \\
\hline 4438 & L1 & $\mathrm{S} 0 / \mathrm{a}$ & 16.8 & 2.66 & $2.34 \times 10^{39}$ & $\ldots$ & 25.40 & $2002 / 01 / 29$ \\
\hline 4457 & L2 & $\mathrm{S} 0 / \mathrm{a}$ & 17.4 & 1.80 & $3.72 \times 10^{39}$ & 7.0 & 39.38 & $2002 / 12 / 03$ \\
\hline 4494 & L2 & E1-2 & 9.7 & 1.52 & $3.47 \times 10^{37}$ & 7.6 & 25.16 & $2001 / 08 / 05$ \\
\hline 4552 & $\mathrm{~T} 2$ & E1 & 16.8 & 2.57 & $3.31 \times 10^{38}$ & 8.5 & 55.14 & $2001 / 04 / 22$ \\
\hline 4636 & L1 & $\mathrm{E} 1 / \mathrm{S} 0$ & 17.0 & 1.81 & $1.86 \times 10^{38}$ & 8.2 & 53.05 & $2000 / 01 / 26$ \\
\hline 5055 & $\mathrm{~T} 2$ & $\mathrm{Sb} / \mathrm{c}$ & 7.2 & 1.32 & $8.13 \times 10^{37}$ & 7.1 & 28.36 & $2001 / 08 / 27$ \\
\hline 5866 & $\mathrm{~T} 2$ & S0 & 15.3 & 1.47 & $1.02 \times 10^{38}$ & 7.7 & 34.18 & $2002 / 11 / 14$ \\
\hline 7331 & $\mathrm{~T} 2$ & $\mathrm{Sb}$ & 14.3 & 8.61 & $3.09 \times 10^{38}$ & 7.6 & 30.13 & $2001 / 01 / 27$ \\
\hline
\end{tabular}

a The coordinates of the nucleus are from isophotal contour fits to 2MASS images, with the following exceptions: NGC 4374 from VLBI observations (Fey et al. 2004), NGC 4438 from VLA observations (Hummel \& Saikia 1991), NGC 4552 from VLBA observations (Nagar et al. 2002), NGC 7331 from stellar kinematics measurements (Argyle \& Clements 1990).

${ }^{\mathrm{b}}$ LINER types, galaxy morphological types, and distances taken from Ho et al. (1997c). LINER types are explained in $\S 2$ of the text.

${ }^{\mathrm{c}}$ Galactic H I column densities from Dickey \& Lockman 1990

${ }^{\mathrm{d}} \mathrm{H} \alpha$ luminosity, taken from Ho et al. (1997a), except NGC 1553, which was taken from Phillips et al. (1986)

e Black hole mass based on the stellar velocity dispersion. See $\S 2$ of the text.

${ }^{\mathrm{f}}$ The emission line strengths reported by Phillips et al. (1986) and Veron-Cetty \& Veron (1986) indicate an $\mathrm{L}$ or $\mathrm{T}$ classification. In the absence of information on broad Balmer lines, we tentatively list this object as $\mathrm{L} 2 / \mathrm{T} 2$. 
TABLE 2

Morphology of the Central Kiloparsec of the Target Galaxies

\begin{tabular}{|c|c|c|c|c|}
\hline $\begin{array}{l}\text { Galaxy } \\
\text { (NGC) }\end{array}$ & $\begin{array}{l}\text { Number } \\
\text { of Point } \\
\text { Sources }\end{array}$ & $\begin{array}{c}\text { Central } \\
\text { Point Source } \\
\text { Properties }^{\mathrm{a}}\end{array}$ & $\begin{array}{l}\text { Diffuse } \\
\text { Emission } \\
\text { Properties }^{\mathrm{a}}\end{array}$ & $\begin{array}{l}\text { Point Source } \\
\text { Sensitivity } \\
\left(\mathrm{erg} \mathrm{s}^{-1}\right)\end{array}$ \\
\hline 1553 & 3 & HS & & $2.6 \times 10^{37}$ \\
\hline 2681 & 3 & HS & $\mathrm{S}$ & $7.6 \times 10^{36}$ \\
\hline 3379 & 12 & $\mathrm{~S}$ & & $7.0 \times 10^{36}$ \\
\hline 3507 & 2 & S & $\mathrm{S}$ & $3.4 \times 10^{37}$ \\
\hline 3607 & 2 & & & $3.4 \times 10^{37}$ \\
\hline 3608 & 2 & S & $\mathrm{S}$ & $4.8 \times 10^{37}$ \\
\hline 3628 & 5 & & $\mathrm{H}$ & $3.4 \times 10^{36}$ \\
\hline 4111 & 1 & $\mathrm{H}$ & $\mathrm{S}$ & $6.6 \times 10^{37}$ \\
\hline 4125 & 1 & HS & & $3.0 \times 10^{37}$ \\
\hline 4314 & 0 & & $\mathrm{~S}$ & $1.9 \times 10^{37}$ \\
\hline 4374 & 4 & HS & $\mathrm{S}$ & $3.3 \times 10^{37}$ \\
\hline 4438 & 1 & HS & $\mathrm{S}$ & $3.8 \times 10^{37}$ \\
\hline 4457 & 1 & HS & $\mathrm{S}$ & $2.6 \times 10^{37}$ \\
\hline 4494 & 6 & $\mathrm{~S}$ & & $1.3 \times 10^{37}$ \\
\hline 4552 & 5 & HS & $\mathrm{S}$ & $1.7 \times 10^{37}$ \\
\hline 4636 & 5 & $\mathrm{~S}$ & $\mathrm{H}$ & $1.8 \times 10^{37}$ \\
\hline 5055 & 5 & HS & & $6.2 \times 10^{36}$ \\
\hline 5866 & 2 & $\mathrm{H}$ & $\mathrm{S}$ & $2.3 \times 10^{37}$ \\
\hline 7331 & 3 & & & $2.3 \times 10^{37}$ \\
\hline
\end{tabular}

${ }^{\mathrm{a}} \mathrm{H}$ indicates that the source is detected in the hard $(2-10 \mathrm{keV})$ band and $\mathrm{S}$ indicates the source is detected in the soft $(0.5-2 \mathrm{keV})$ band.

b The lowest detectable $0.5-10 \mathrm{keV}$ point source luminosity in a given observation (assuming an absorbed power-law spectrum with $\Gamma=1.8$, and $N_{\mathrm{H}}=2 \times 10^{20} \mathrm{~cm}^{-2}$; see $\left.\S 3.2\right)$. 
TABle 3

Spectral Models and Parameters for Bright Sources

\begin{tabular}{|c|c|c|c|}
\hline $\begin{array}{l}\text { Galaxy } \\
(\mathrm{NGC})\end{array}$ & Best-Fitting Models and Parameters ${ }^{a}$ & $\chi^{2} /$ d.o.f. & $\begin{array}{c}\text { Unabsorbed } \\
L_{0.5-10 \mathrm{keV}} \\
\left(\mathrm{erg} \mathrm{s}^{-1}\right)\end{array}$ \\
\hline \multicolumn{4}{|c|}{ Central Point Sources } \\
\hline 1553 & $\begin{array}{l}\text { mekal2T, } \quad N_{\mathrm{H}}=5_{-1}^{+2} \times 10^{21} \mathrm{~cm}^{-2}, k T_{1}=0.14_{-0.09}^{+0.04} \mathrm{keV}, k T_{2}=18_{-10}^{+70} \mathrm{keV} \\
\text { pow } \quad N_{\mathrm{H}}=3.2^{+0.8} \times 10^{21} \mathrm{~cm}^{-2} \Gamma_{\Gamma=1.2^{+0.2}}\end{array}$ & $\begin{array}{c}51.76 / 40 \\
63.6 / 43\end{array}$ & $\begin{array}{l}1.5 \times 10^{40} \\
1.6 \times 10^{40}\end{array}$ \\
\hline 2681 & 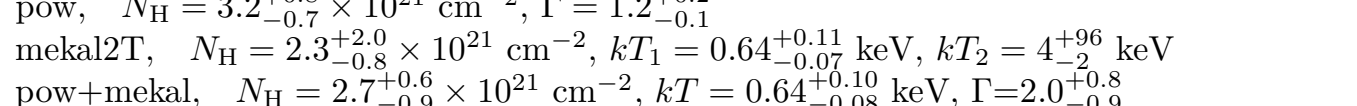 & $\begin{array}{l}63.6 / 43 \\
8.3 / 14 \\
8.2 / 14\end{array}$ & $\begin{array}{l}1.6 \times 10^{20} \\
9.0 \times 10^{38} \\
9.9 \times 10^{38}\end{array}$ \\
\hline 4374 & $\begin{array}{l}\text { mekal }+ \text { pow }, \quad N_{\mathrm{H}}=2.0_{-0.6}^{+7.9} \times 10^{21} \mathrm{~cm}^{-2}, k T=0.6_{-0.4}^{+0.5} \mathrm{keV}, \Gamma=2.0_{-0.1}^{+0.2} \\
\text { pow, } \quad N_{\mathrm{H}}=1.9 \pm 0.5 \times 10^{21} \mathrm{~cm}^{-2}, \Gamma=2.1 \pm 0.2\end{array}$ & $\begin{array}{l}14.6 / 27 \\
18.4 / 29\end{array}$ & $\begin{array}{l}7.7 \times 10^{39} \\
7.5 \times 10^{39}\end{array}$ \\
\hline 4438 & $\begin{array}{l}\text { mekal+pow }, \quad N_{\mathrm{H}}=1.2 \pm 0.9 \times 10^{21} \mathrm{~cm}^{-2}, k T=0.8 \pm 0.6 \mathrm{keV}, \Gamma=1.1 \pm 1.1 \\
\text { mekal, } \quad N_{\mathrm{H}}=3_{-3}^{+2} \times 10^{21} \mathrm{~cm}^{-2}, k T=0.8_{-0.2}^{+0.1} \mathrm{keV}\end{array}$ & $\begin{array}{l}9.3 / 12 \\
8.4 / 14\end{array}$ & $\begin{array}{l}4.0 \times 10^{39} \\
9.4 \times 10^{38}\end{array}$ \\
\hline 4457 & $\begin{array}{l}\text { mekal+pow }, \quad N_{\mathrm{H}}=9.8 \pm 0.1 \times 10^{20} \mathrm{~cm}^{-2}, k T=0.6 \pm 0.8 \mathrm{keV}, \Gamma=1.7 \pm 0.3 \\
\text { mekal2T }, \quad N_{\mathrm{H}}=8 . ._{-6}^{+8} \times 10^{20} \mathrm{~cm}^{-2}, k T_{1}=0.7_{-0.2}^{+0.3} \mathrm{keV}, k T_{2}=7_{-3}^{+42} \mathrm{keV}\end{array}$ & $\begin{array}{l}6.1 / 9 \\
8.8 / 9\end{array}$ & $\begin{array}{l}2.1 \times 10^{39} \\
1.8 \times 10^{39}\end{array}$ \\
\hline 4494 & $\begin{array}{l}\text { mekal2T, } \quad N_{\mathrm{H}}=5.13_{-5.1}^{+630} \times 10^{19} \mathrm{~cm}^{-2}, k T_{1}=0.7_{-0.4}^{+0.6} \mathrm{keV}, k T_{2}=6_{-2}^{+13} \mathrm{keV} \\
\text { pow } \quad N_{\mathrm{H}}=3_{-3}^{+7} \times 10^{20} \mathrm{~cm}^{-2}, \Gamma=1.8 \pm 0.3\end{array}$ & $\begin{array}{l}13.2 / 14 \\
18.6 / 16\end{array}$ & $\begin{array}{l}1.6 \times 10^{39} \\
1.5 \times 10^{39}\end{array}$ \\
\hline 4552 & $\begin{array}{l}\text { pow, } \quad N_{\mathrm{H}}=6_{-6}^{+6} \times 10^{20} \mathrm{~cm}^{-2}, \Gamma=2.0 \pm 0.2 \\
\text { compbb, } \quad N_{\mathrm{H}}=1.58 \pm 0.04 \times 10^{20} \mathrm{~cm}^{-2}, k T=(9.06 \pm 0.07) \times 10^{-2} \mathrm{keV}, \tau=7.03_{-0.03}^{+0.01}\end{array}$ & $\begin{array}{l}43.7 / 43 \\
42.5 / 42\end{array}$ & $\begin{array}{l}3.7 \times 10^{39} \\
3.7 \times 10^{39}\end{array}$ \\
\hline 5055 & mekal2T, $\quad N_{\mathrm{H}}=0.5_{-0.5}^{+0.4} \times 10^{22} \mathrm{~cm}^{-2}, k T_{1}=0.2_{-0.2}^{+8} \mathrm{keV}, k T_{2}=9_{-2}^{+70} \mathrm{keV}$ & $4.3 / 10$ & $8.3 \times 10^{38}$ \\
\hline \multicolumn{4}{|c|}{ Average Diffuse Emission Spectra } \\
\hline $\begin{array}{l}\text { ellipticals } \\
\text { spirals }\end{array}$ & $\begin{array}{ll}\text { mekal+pow }, & N_{\mathrm{H}}=9_{-8}^{+31} \times 10^{19} \mathrm{~cm}^{-2}, k T=0.56_{-0.03}^{+0.02} \mathrm{keV}, \Gamma=1.3_{-0.2}^{+0.1} \\
\text { mekal+pow }, & N_{\mathrm{H}}=(7 \pm 3) \times 10^{20} \mathrm{~cm}^{-2}, k T=0.57_{-0.04}^{+0.02} \mathrm{keV}, \Gamma=1.5_{-0.1}^{+0.3}\end{array}$ & $\begin{array}{l}208.4 / 177 \\
237.1 / 236\end{array}$ & $\begin{array}{l}5.3 \times 10^{38} \\
8.5 \times 10^{38}\end{array}$ \\
\hline \multicolumn{4}{|c|}{ Central Diffuse Emission Patches of Individual Galaxies } \\
\hline 3628 & $\begin{array}{l}\text { mekal2T, } \quad N_{\mathrm{H}}=6_{-2}^{+5} \times 10^{21} \mathrm{~cm}^{-2}, k T_{1}=0.2_{-0.1}^{+0.4} \mathrm{keV}, k T_{2}=7_{-4}^{+19} \mathrm{keV} \\
\text { pow } \quad N_{\mathrm{H}}=2_{-1}^{+2} \times 10^{21} \mathrm{~cm}^{-2}, \Gamma=1.5_{-0.2}^{+0.3}\end{array}$ & $\begin{array}{l}18.07 / 18 \\
21.26 / 20\end{array}$ & $\begin{array}{l}4.6 \times 10^{38} \\
4.6 \times 10^{38}\end{array}$ \\
\hline \multicolumn{4}{|c|}{ Brightest Off-Nuclear Point Sources of Galaxies Without a Central Source } \\
\hline 3379 & $\begin{array}{l}\text { pow }, \quad N_{\mathrm{H}}=1.1_{-1.0}^{+0.7} \times 10^{21} \mathrm{~cm}^{-2}, \Gamma=2.1_{-0.2}^{+0.3} \\
\text { mekal }+ \text { pow }, \quad N_{\mathrm{H}}=1.8_{-0.8}^{+1.8} \times 10^{21} \mathrm{~cm}^{-2}, k T=0.08_{-0.01}^{+0.14} \mathrm{keV}, \Gamma=2.3_{-0.3}^{+0.5}\end{array}$ & $\begin{array}{l}31.8 / 27 \\
27.2 / 25\end{array}$ & $\begin{array}{l}1.3 \times 10^{39} \\
1.5 \times 10^{39}\end{array}$ \\
\hline
\end{tabular}

a The two best-fitting models for sources with more than 200 counts in their spectra. In the case of NGC 5055, only one reasonable, well-fitting model was found. See $\S 3.2$ for explanation of models and their parameters. 
TABle 4

Diffuse Emssion Luminsity and Candidate AGN Properties

\begin{tabular}{|c|c|c|c|c|c|c|c|c|}
\hline \multirow{2}{*}{$\begin{array}{l}\text { Galaxy } \\
(\mathrm{NGC})\end{array}$} & \multicolumn{2}{|c|}{ Diffuse, 1 kpc Region ${ }^{\mathrm{a}}$} & \multicolumn{2}{|c|}{ Diffuse, $2^{\prime \prime} 5$ Region $^{\mathrm{a}}$} & \multicolumn{4}{|c|}{ Candidate AGN $^{\mathrm{a}}$} \\
\hline & $0.5-2 \mathrm{keV}$ & $2-10 \mathrm{keV}$ & $0.5-2 \mathrm{keV}$ & $2-10 \mathrm{keV}$ & $0.5-2 \mathrm{keV}$ & $2-10 \mathrm{keV}$ & $\mathcal{R}_{\text {Edd }}{ }^{\mathrm{b}}$ & $\log R_{\mathrm{X}}{ }^{\mathrm{c}}$ \\
\hline 1553 & $3.3 \times 10^{38}$ & $1.7 \times 10^{38}$ & $1.6 \times 10^{38}$ & $8.4 \times 10^{37}$ & $3.0 \times 10^{39}$ & $1.2 \times 10^{40}$ & $8.4 \times 10^{-6}$ & -2.7 \\
\hline 2681 & $1.3 \times 10^{39}$ & $7.1 \times 10^{38}$ & $9.9 \times 10^{38}$ & $5.1 \times 10^{38}$ & $1.6 \times 10^{38}$ & $1.8 \times 10^{38}$ & $8.0 \times 10^{-7}$ & -1.9 \\
\hline 3379 & $4.3 \times 10^{38}$ & $2.2 \times 10^{38}$ & $1.1 \times 10^{38}$ & $5.6 \times 10^{37}$ & $4.6 \times 10^{37}$ & $1.7 \times 10^{37}$ & $6.0 \times 10^{-9}$ & -2.2 \\
\hline 3507 & $7.8 \times 10^{38}$ & $4.0 \times 10^{38}$ & $6.4 \times 10^{38}$ & $3.3 \times 10^{38}$ & $<2.5 \times 10^{37}$ & $<3.9 \times 10^{37}$ & & \\
\hline 3607 & $9.7 \times 10^{37}$ & $5.0 \times 10^{37}$ & $9.1 \times 10^{37}$ & $4.7 \times 10^{37}$ & $<3.1 \times 10^{37}$ & $<5.0 \times 10^{37}$ & $<1.4 \times 10^{-8}$ & \\
\hline 3608 & $8.7 \times 10^{37}$ & $4.5 \times 10^{37}$ & $8.3 \times 10^{37}$ & $4.3 \times 10^{37}$ & $2.7 \times 10^{38}$ & $6.0 \times 10^{38}$ & $3.3 \times 10^{-7}$ & $<-2.7$ \\
\hline 3628 & $1.1 \times 10^{39}$ & $5.5 \times 10^{38}$ & $8.9 \times 10^{38}$ & $4.6 \times 10^{38}$ & $<3.1 \times 10^{36}$ & $<5.0 \times 10^{36}$ & $<4.4 \times 10^{-9}$ & \\
\hline 4111 & $1.3 \times 10^{39}$ & $7.0 \times 10^{38}$ & $8.7 \times 10^{38}$ & $4.5 \times 10^{38}$ & $7.0 \times 10^{38}$ & $3.7 \times 10^{39}$ & $4.1 \times 10^{-6}$ & -3.0 \\
\hline 4125 & $2.9 \times 10^{38}$ & $1.5 \times 10^{38}$ & $1.7 \times 10^{38}$ & $9.2 \times 10^{37}$ & $3.4 \times 10^{38}$ & $5.4 \times 10^{38}$ & $1.5 \times 10^{-7}$ & $<-2.7$ \\
\hline 4314 & $2.3 \times 10^{39}$ & $1.2 \times 10^{39}$ & $2.3 \times 10^{38}$ & $1.2 \times 10^{38}$ & $<1.8 \times 10^{37}$ & $<2.9 \times 10^{37}$ & $<1.3 \times 10^{-7}$ & \\
\hline 4374 & $2.5 \times 10^{39}$ & $1.3 \times 10^{39}$ & $1.3 \times 10^{39}$ & $6.6 \times 10^{38}$ & $3.4 \times 10^{39}$ & $4.0 \times 10^{39}$ & $4.4 \times 10^{-7}$ & -1.3 \\
\hline 4438 & $3.7 \times 10^{39}$ & $1.9 \times 10^{39}$ & $3.9 \times 10^{38}$ & $2.0 \times 10^{38}$ & $2.6 \times 10^{39}$ & $1.2 \times 10^{39}$ & & \\
\hline 4457 & $6.2 \times 10^{38}$ & $3.2 \times 10^{38}$ & $4.2 \times 10^{38}$ & $2.2 \times 10^{38}$ & $5.3 \times 10^{38}$ & $9.7 \times 10^{38}$ & $6.8 \times 10^{-6}$ & -1.6 \\
\hline 4494 & $7.6 \times 10^{38}$ & $3.9 \times 10^{38}$ & $7.2 \times 10^{38}$ & $3.7 \times 10^{38}$ & $6.3 \times 10^{38}$ & $9.9 \times 10^{38}$ & $1.7 \times 10^{-6}$ & $<-3.7$ \\
\hline 4552 & $1.7 \times 10^{39}$ & $8.8 \times 10^{38}$ & $1.2 \times 10^{39}$ & $6.1 \times 10^{38}$ & $1.7 \times 10^{39}$ & $2.0 \times 10^{39}$ & $4.4 \times 10^{-7}$ & -1.5 \\
\hline 4636 & $7.7 \times 10^{38}$ & $4.0 \times 10^{38}$ & $5.4 \times 10^{38}$ & $2.8 \times 10^{38}$ & $<3.9 \times 10^{38}$ & $<6.2 \times 10^{38}$ & $<2.7 \times 10^{-7}$ & \\
\hline 5055 & $1.6 \times 10^{39}$ & $8.3 \times 10^{38}$ & $4.5 \times 10^{38}$ & $2.3 \times 10^{38}$ & $<1.3 \times 10^{38}$ & $<2.0 \times 10^{38}$ & $<1.1 \times 10^{-6}$ & \\
\hline 5866 & $2.1 \times 10^{38}$ & $1.1 \times 10^{38}$ & $5.4 \times 10^{37}$ & $2.8 \times 10^{37}$ & $1.0 \times 10^{37}$ & $3.1 \times 10^{38}$ & $4.3 \times 10^{-7}$ & -1.5 \\
\hline 7331 & $6.6 \times 10^{38}$ & $3.4 \times 10^{38}$ & $4.2 \times 10^{38}$ & $2.2 \times 10^{38}$ & $<2.1 \times 10^{37}$ & $<3.4 \times 10^{37}$ & $<6.0 \times 10^{-8}$ & \\
\hline
\end{tabular}

${ }^{\text {a }}$ Luminosities are given in $\operatorname{erg~s}^{-1}$.

b The Eddington ratio; see equation (4) in $\S 6$.

c The X-ray radio-loudness parameter; see equation (3) in $\S 5$. 
For a complete preprint, including images, see http://www.astro.psu.edu/users/mce/preprints/

FIG. 1.- Montage of the Chandra images of the target galaxies. For each galaxy the image encompass 1 kpc in radius centered on the center of the galaxy. The size of each pixel is $00^{\prime \prime} 5$. For each target, the left image is for the energy band $0.5-2$ keV, the middle image is for the energy band 2-10 keV and the right image is the hardness ratio map (see §3). The grey scale represents the number of counts per pixel and is logarithmic and adjusted to give the maximal dynamical range for each image. North is up and east is to the left. 

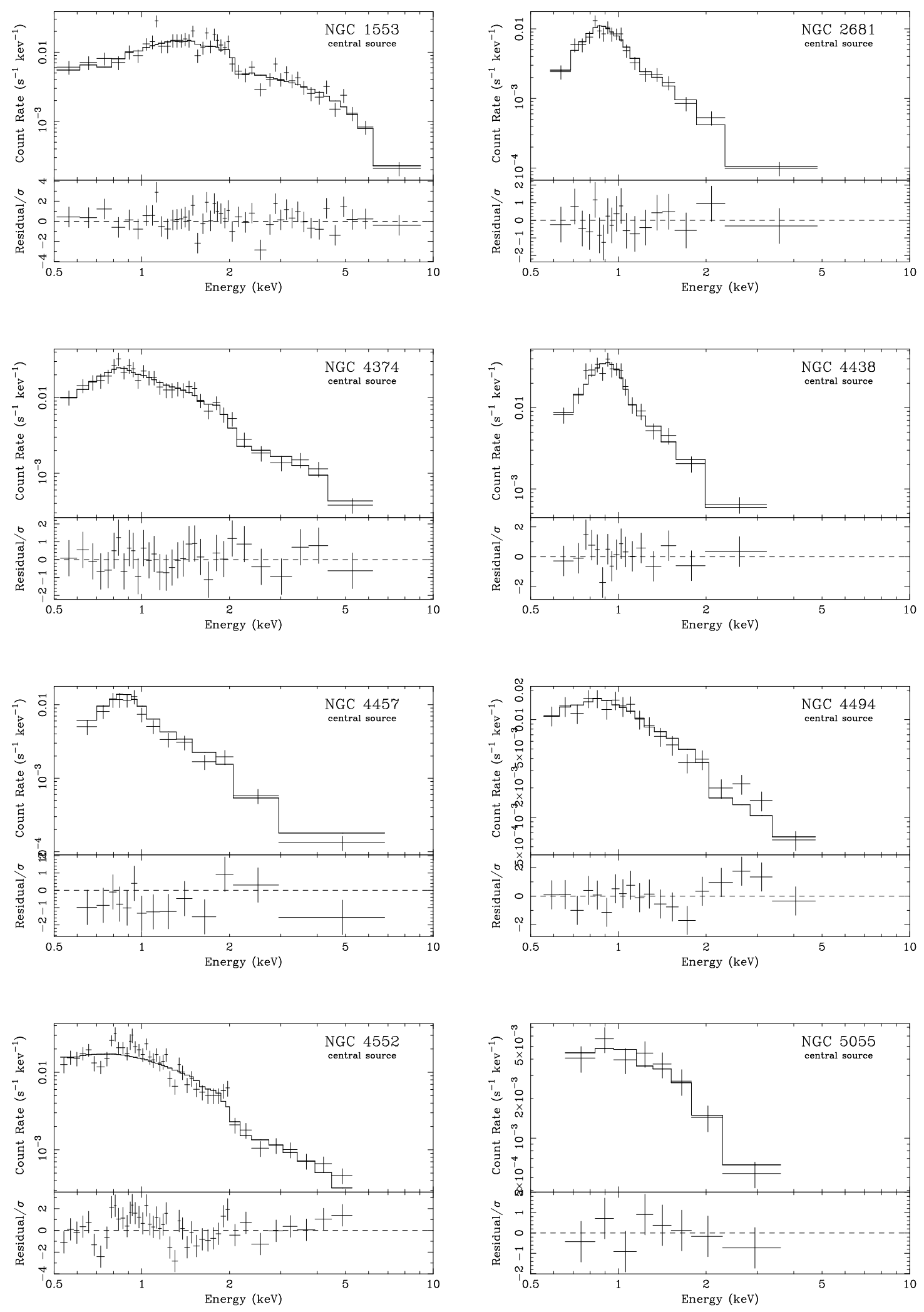

Fig. 2.- Spectra of the sources listed in Table 3 with the best-fitting model superposed. The lower frame shows the residuals scaled by the error bars. The models and their parameters are given in Table 3. 

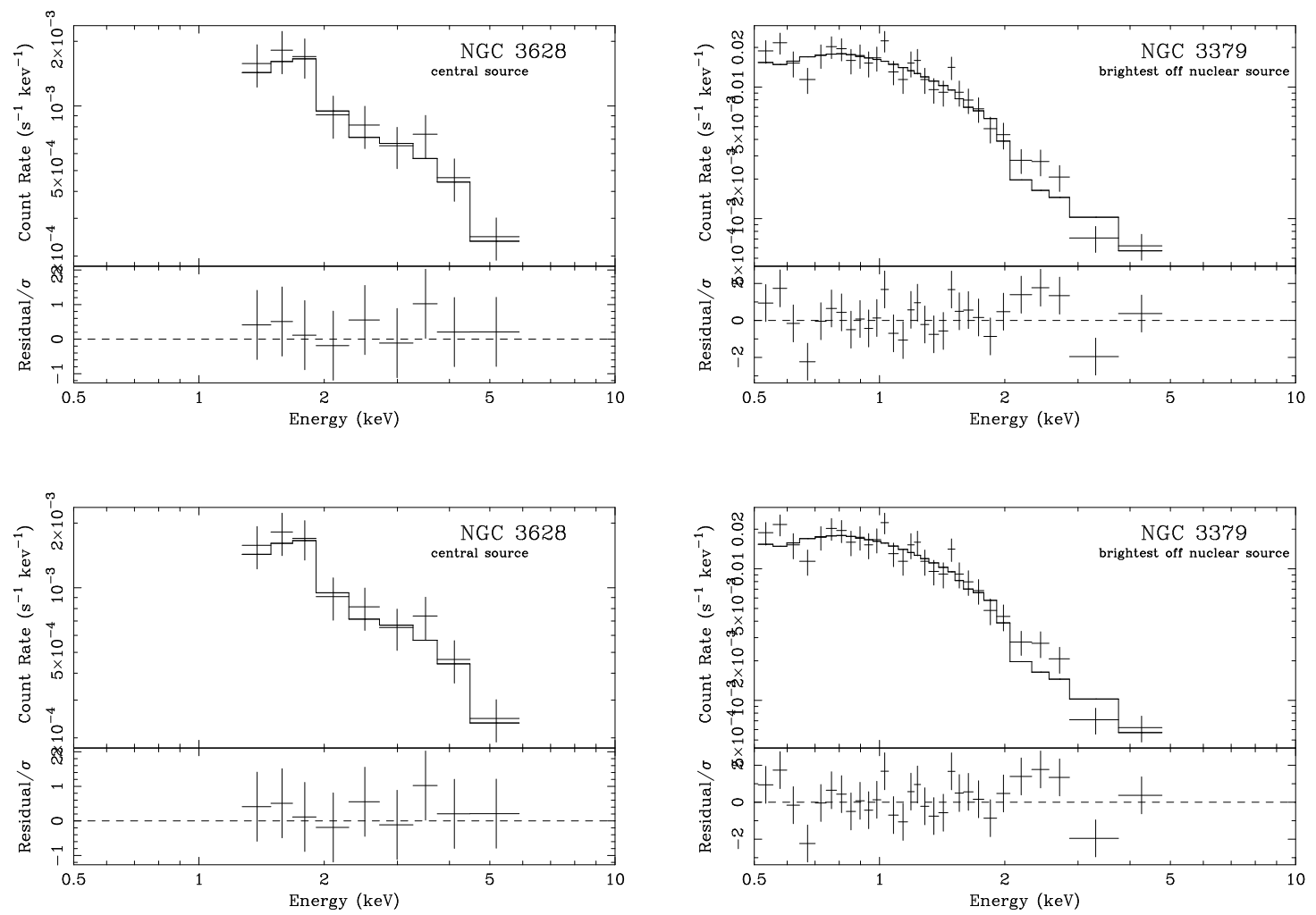

Figure 2 - continued.
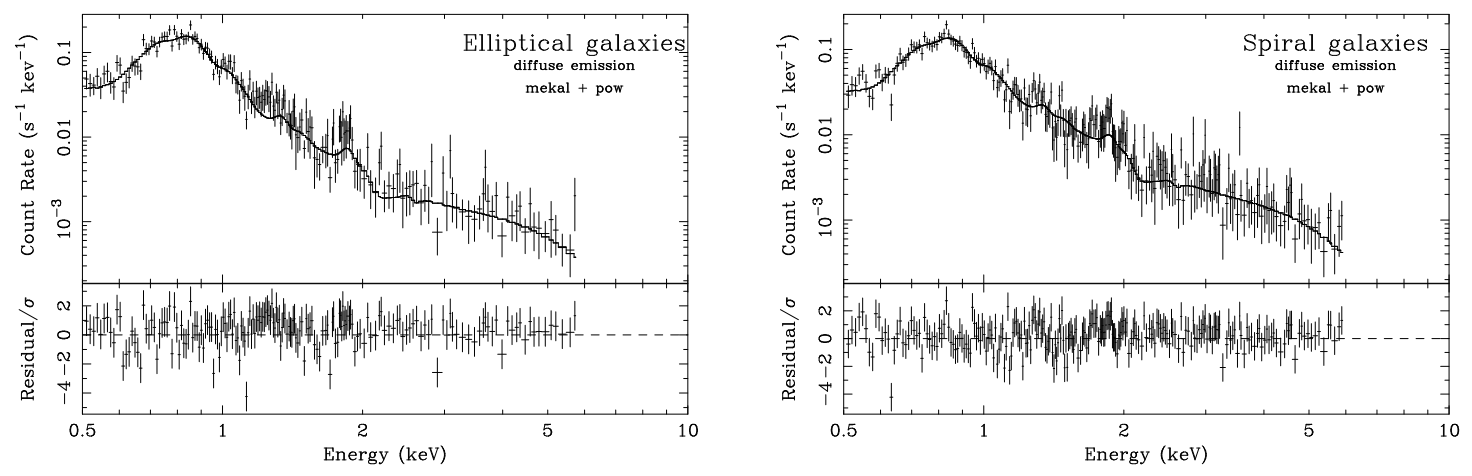

FIG. 3.- Stacked diffuse emission spectra for the elliptical galaxies (left panel) and the spiral galaxies( right panel). Details of the models fitted to the data are given in $\S 6$ of the text. 

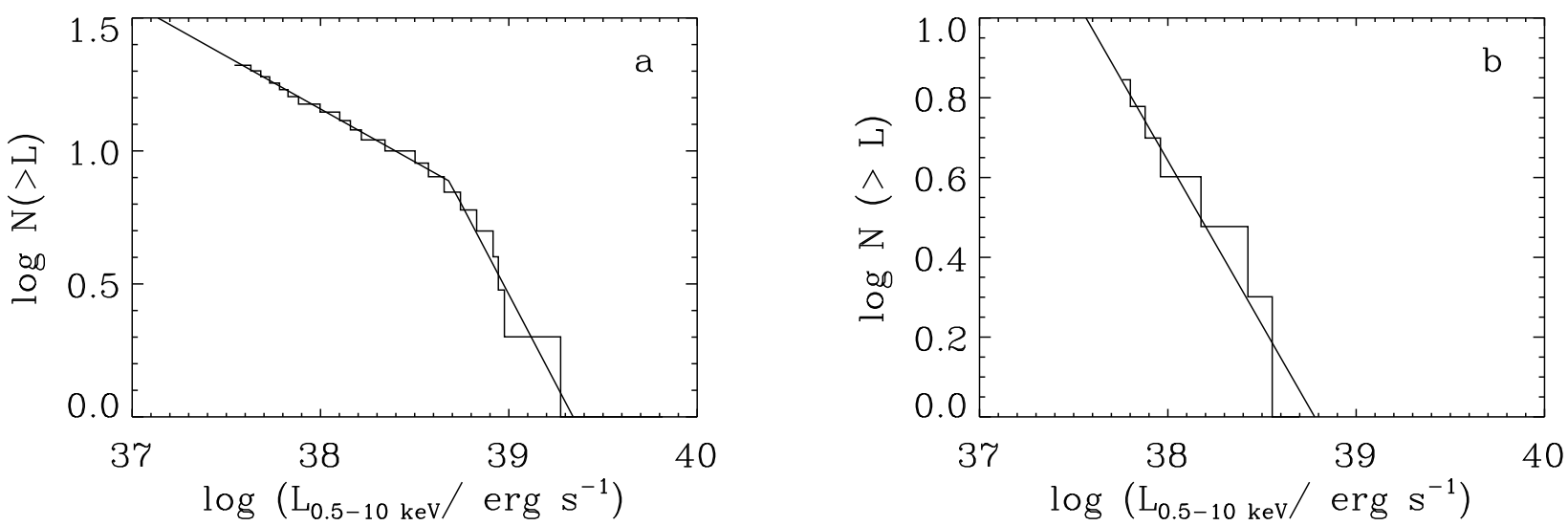

FIG. 4.- Histograms showing the cumulative luminosity functions for the point sources in all the elliptical (a) and all the spiral galaxies (b). The continuous lines show single or broken power-law fits to the data (see $\S 5$ of the text for full details).
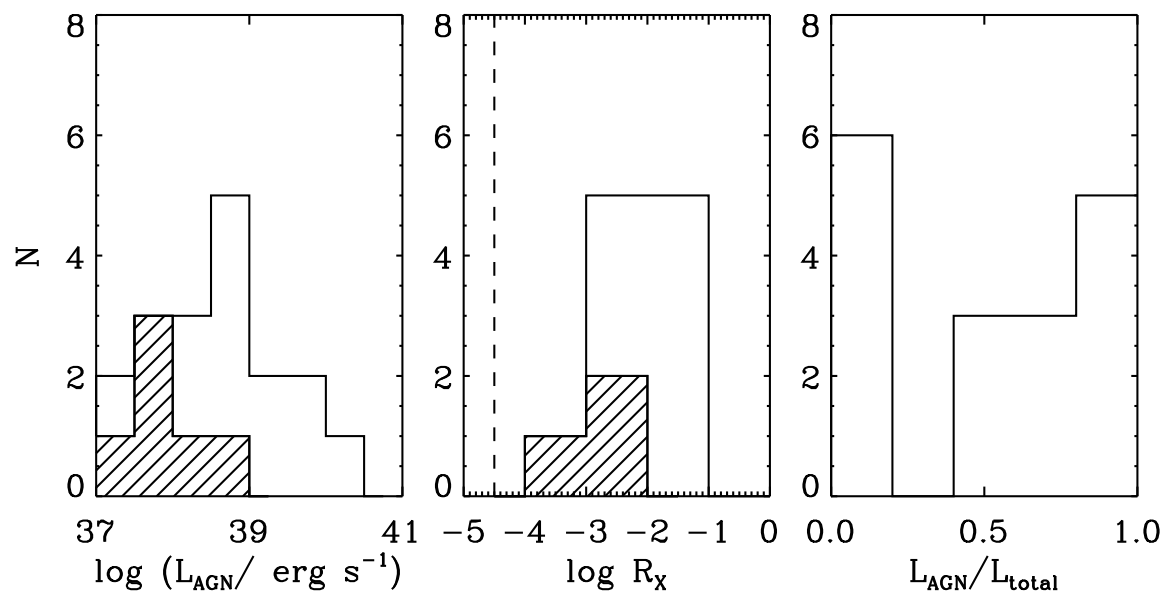

FIG. 5.- Histograms showing the distribution of $L_{2-10 \mathrm{keV}}^{\mathrm{AGN}}, \log R_{X}$, and $L_{0.5-10 \mathrm{keV}}^{\mathrm{AGN}} / L_{0.5-10 \mathrm{keV}}^{2 ! \prime}$ for the galaxies of our sample. The hatched regions represent upper limits. The threshold between radio-loud and radio-quiet sources is shown on the log $R_{X}$ histogram as a dashed line.
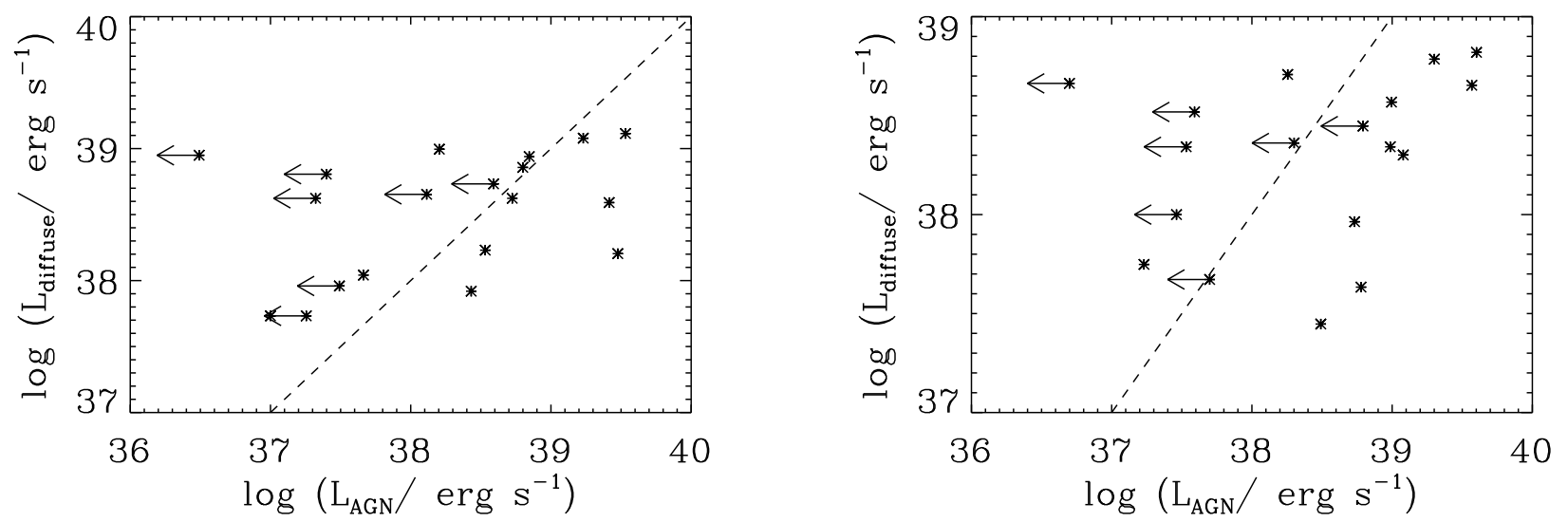

FIG. 6. - Luminosity from the diffuse emission in the inner $2 . \prime 5$ as a function of the luminosity from the AGN in the $0.5-2 \mathrm{keV}$ band (top) and $2-10 \mathrm{keV}$ band (bottom). The dashed line represents equal luminosity from the diffuse emission equals and from the AGN. 


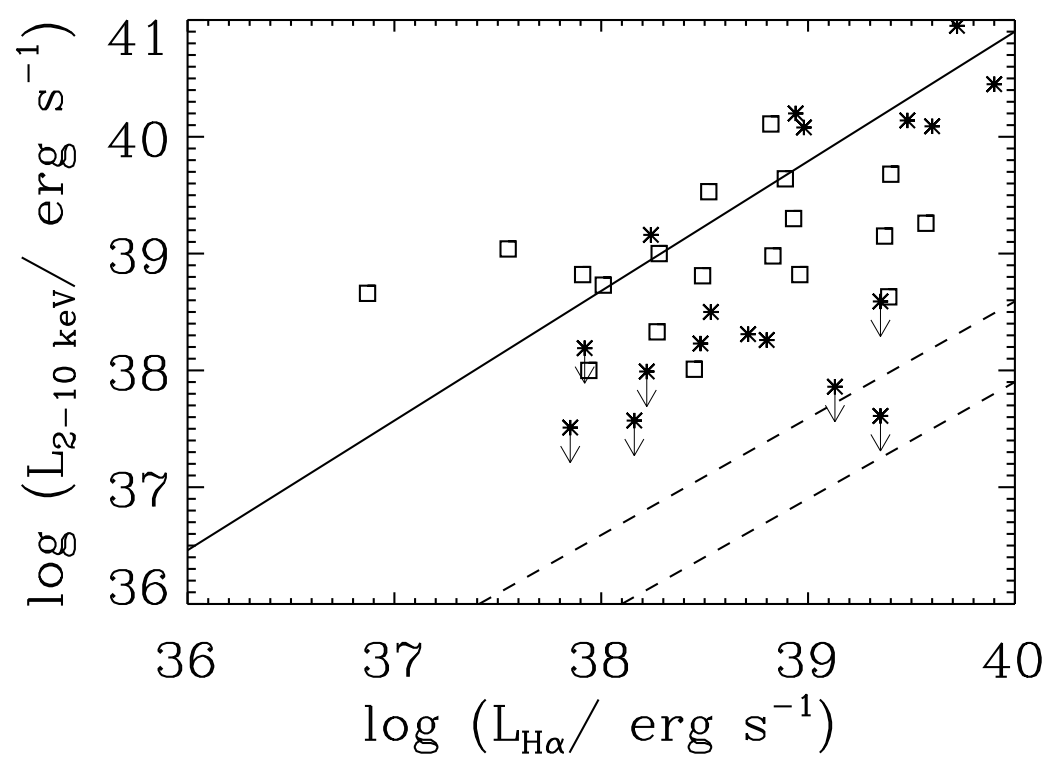

FIG. 7.- $L_{2-10 \mathrm{keV}}-L_{H \alpha}$ plot for our sample (squares) and the objects from the Ho et al. (2001) sample (stars). The X-ray luminosity is measured within the inner 2 ' 5 region. The solid line represents the best fit found by Ho et al. (2001) for low-redshift quasars and Seyfert 1 galaxies. The dotted lines represent the range of luminosities where a starburst would fall.
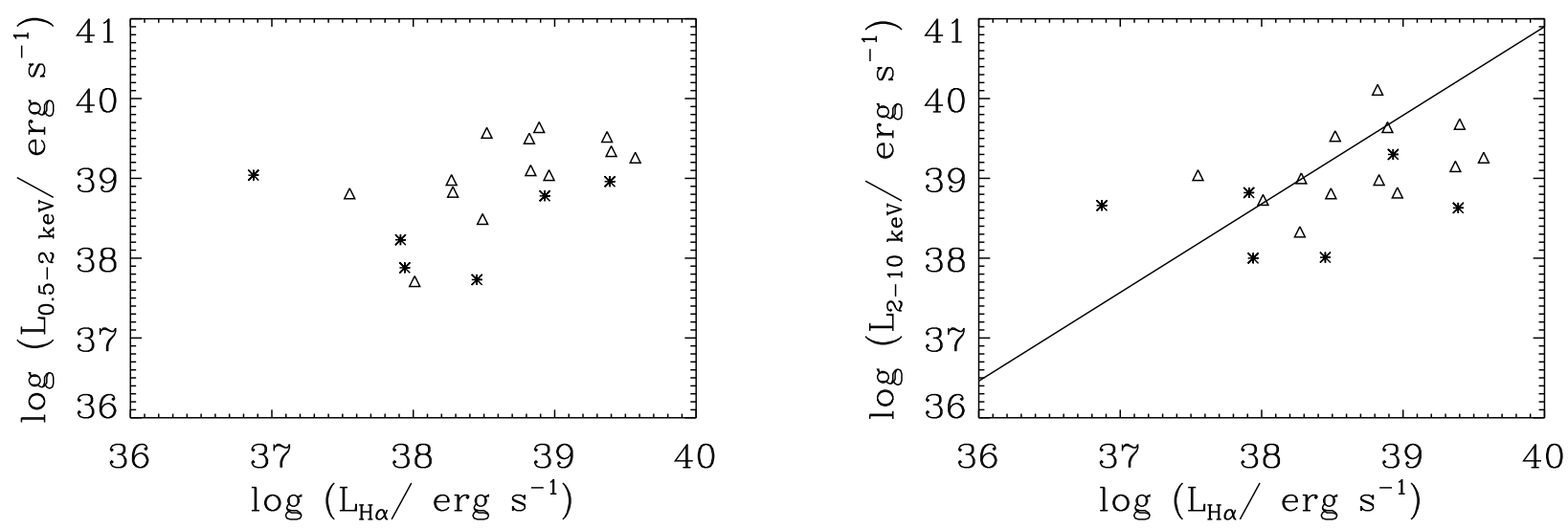

FIG. 8.- $L_{0.5-2 \mathrm{keV}}-L_{H \alpha}$ and $L_{2-10 \mathrm{keV}}-L_{H \alpha}$ diagrams. The stars represent LINERs whose X-ray luminosity of the central 2 '. 5 region is dominated by diffuse emission while triangles represent AGN-dominated LINERs. The solid line shows the extrapolation of the correlation found for low-redshift quasars and Seyfert 1 galaxies (from Ho et al. 2001). 
This figure "f1a.jpg" is available in "jpg" format from: http://arxiv.org/ps/astro-ph/0604487v1 
This figure "f1b.jpg" is available in "jpg" format from: http://arxiv.org/ps/astro-ph/0604487v1 
This figure "f1c.jpg" is available in "jpg" format from: http://arxiv.org/ps/astro-ph/0604487v1 
This figure "f1d.jpg" is available in "jpg" format from: http://arxiv.org/ps/astro-ph/0604487v1 
This figure "f1e.jpg" is available in "jpg" format from: http://arxiv.org/ps/astro-ph/0604487v1 